NBER WORKING PAPER SERIES

THE TRANSFER OF HUMAN CAPITAL

Boyan Jovanovic

Yaw Nyarko

Working Paper No. 4823

\author{
NATIONAL BUREAU OF ECONOMIC RESEARCH \\ 1050 Massachusetts Avenue \\ Cambridge, MA 02138 \\ August 1994
}

We thank the C.V. Starr Center for Applied Economics at New York University for technical and financial help, and Jacob Mincer and Nachum Sicherman for useful comments, and Chung Tse for help with the research. This paper is part of NBER's research program in Productivity. Any opinions expressed are those of the authors and not those of the National Bureau of Economic Research.

( ) 1994 by Boyan Jovanovic and Yaw Nyarko. All rights reserved. Short sections of text, not to exceed two paragraphs, may be quoted without explicit permission provided that full credit, including $(-)$ notice, is given to the source. 
NBER Working Paper \#4823

August 1994

\title{
THE TRANSFER OF HUMAN CAPITAL
}

\begin{abstract}
Most of our productive knowledge was handed down to us by previous generations. The transfer of knowledge from the old to the young is therefore a comerstone of productivity growh. We study this process in a model in which the old sell knowledge to the young -- old workers train the young, and charge them for this service. We take an information-theoretic approach in which training occurs if a young agent watches an old worker perform a task. This assumption has plenty of empirical support -- in their first three months on the job, young workers spend about five times as long watching others work as they do in formal training programs.

Equilibrium is not constrained Pareto optimal. The old have private information, and this gives rise to an adverse selection problem: some old agents manage to sell skills that the young would not buy (if only they knew exactly what they were buying). We derive the implications for the lifetime of technological lines, and we show that the model generates a negative relation between a firm's productivity and its probability of failure.
\end{abstract}

Boyan Jovanovic Department of Economics New York University 269 Mercer Street New York, NY 10003 and NBER
Yaw Nyarko

Department of Economics

New York University

269 Mercer Street

New York, NY 10003 
Jovanovic and Nyarko The Transfer of Human Capital

\section{Introduction}

How efficiently is productive knowledge passed on from one generation to the next? Some of this knowledge is handed down informally in the family, and some of it is taught in schools. A third mechanism is learning on the job: acquiring productive knowledge through the process of on-the-job training and learning by doing. Our paper focuses on the third mechanism.

Two prominent equilibrium models of leaming on the job are those of Prescott and Boyd (1987), and Chari and Hopenhayn (1991). They assume that the process by which one worker learns from other workers can be (and is) fully internalized by firms . Our approach is similar in spirit, but our conclusions differ from theirs. We explicitly model the information that the process of training transfers, and this leads to the conclusion that the market for on-the-job training is plagued by the efficiency problem that usually crops up with markets for information.

We study an overlapping generations economy in which people live for two periods. An old worker trains the young worker who works alongside him. This training consists of an explicit mechanism by which information is revealed to the young worker. The information consists of observations of past signals about the unknown parameters of the production process that the old and the young worker alike both use. So although agents die, their information can survive for ever.

There are two fundamental differences between this economy and a frictionless one in which a pair of infinitely lived workers were to share their information instantaneously; both of them imply a level of output that is lower in the overlapping generations economy than in the infinite horizon one. The first difference occurs because the transfer of knowledge is assumed to take time: the young worker must watch the old worker $d c$ the jobl. Although the young worker can work and watch at the same time (so that training is assumed to involve no foregone output cost), information still accumulates more slowly than it would if an infinitely lived agents were to observe the same signals.

\footnotetext{
${ }^{1}$ The assumption that the transfer of knowledge occurs through watching is backed by a study by Barron, Black and Loewenstein (1989) who report (in table 1) that in a sample of some 2,000 employers, the typical worker spent at least five times as many hours watching others work as he did in formal training programs.
} 
Jovenovic and Nyarko The Transfer of Human Capital

This is because there is no way for the old worker to pass on the information that he gains from the signal that he sees in the second period of his life. Therefore, in the overlapping generations economy, the two-worker team will have only half the productive information that a pair of infinitely lived individuals would have, and a permanently lower level of output around trend. This first difference therefore simply reflects the added constraints that we impose on informational transfer among people.

The second difference stems from a failure in the market for information. We follow Prescott, Boyd, Chari, and Hopenhayn assume that the old sell training to the young. The content of the training is known to the old worker, but not to a prospective young buyer. The young worker therefore enters a match with an old worker based on what he knows about the population of all old workers. Old workers with inferior skills exploit this, and in equilibrium too much training occurs in comparison to what a central planner could achieve through a tax scheme. Equilibrium allows the survival of some skills that the planner would prefer to extinguish so as to release more young agents to start new technologies. In this sense, competitive equilibrium involves too much resistance to change.

\section{Model}

There will be many technological lines. We first describe what happens within one such line.

2.1. Within a Technological "Line:" A technological line has many grades indexed by $n=0,1,2, \ldots$ The production function and information structure within a technological line are taken from Jovanovic and Nyarko (1994). If an agent uses grade $n$ at date $t$, his decision $z$ yields net output $q$ via the production function

$$
q=\gamma^{n}\left[1-\left(y_{n t}-z\right)^{2}\right]
$$

Here $y_{n}$ is a random variable that acts as an unknown "target", and is observed after $z$ is chosen. 
Jovanovic and Nyarko The Transfer of Human Capizal

Since $\gamma \geq 1$, a larger $n$ denotes a better grade. Since $y_{n t}$ is observed regardless of what $z$ is chosen, $z$ does not affect learning. Assume that everyone is risk-neutral, so that each agent maximizes his own expected output. Let $E_{1}(\cdot)$ denote the conditional expectation at date $t$. The decision that maximizes $E_{\imath}(q)$ in (1) is

$$
z=E_{t}\left(y_{0}\right)
$$

The random target fluctuates around a grade-specific parameter $\theta_{\mathrm{n}}$ :

$$
y_{n 1}=\theta_{n}+w_{n 1}
$$

The agent does not know $\theta_{a}$. He can observe $y_{a}$ but only if at date $t$ he uses grade $n$. Let $N\left(\mu, \sigma^{2}\right)$ denote the normal distribution with mean $\mu$ and variance $\sigma^{2}$. Assume that $w_{n t}$ is an i.i.d variate, with marginal distribution $N\left(0, \sigma_{w}{ }^{2}\right)$. Since $E_{t}\left(w_{n}\right)=0$, equation (2) implies that the optimal decision is

$$
z=E_{i}\left(\theta_{n}\right)
$$

and (1) - (4) imply that expected net output is

$$
E_{1}(q)=\gamma^{n}\left[1-\operatorname{Var}_{r}\left(\theta_{n}\right)-\sigma_{w}{ }^{2}\right]
$$

where $\operatorname{Var}_{1}(\cdot)$ denotes the conditional variance. If he uses grade $n$, he observes $y_{n 1}$ and leams more about $\theta_{n}$, which allows him to make a better decision $z$. This reduces $\operatorname{Var}_{n}\left(\theta_{n}\right)$, and raises his expected net output. Indeed, suppose that the agent has a prior belief over $\theta_{a}$ which is $N\left(m_{a}, x_{a}\right)$ where

$$
m_{n}=E_{t} \theta_{n} \quad \text { and } \quad x_{n}=v^{2} \theta_{t} \theta_{n}
$$


Jovanovic and Nyorko The Transter of Human Capital

Then upon observing $y_{n}$ it is easy to verify that the updated beliefs over $\theta_{n}$ will be normal with variance given by the function

$$
h_{1}(x) \equiv \sigma_{w}{ }^{2} x /\left(\sigma_{w}{ }^{2}+x\right)
$$

in particular the posterior variance is $x_{n}^{+}=h_{1}\left(x_{n}\right)$. The updated posterior mean is given by

$$
m_{n}=h_{1}\left(x_{n}\right)\left\{m_{n} / x_{n}+y_{n} / a_{w}^{2}\right\}
$$

However this learning process is bounded: Using grade $n$ forever allows the agent to leam $\theta_{n}$ completely so that $E_{i}(q) \rightarrow \gamma^{n}\left[1-\sigma_{w}{ }^{2}\right]<\infty$.

2.2. Upgrading Within a Technological Line: Successive grades are linked as follows:

$$
\theta_{n+1}=\sqrt{\alpha} \theta_{a} \cdot \epsilon_{a, 1}, \quad n=1, \ldots .
$$

The $\epsilon$ 's are iid with distribution $N\left(0, \sigma_{e}^{2}\right), \epsilon_{n+1}$ and $\theta_{\mathrm{a}}$ are independent of each other. Agents know $\alpha$ and $\sigma_{\epsilon}{ }^{2}$, but they do not see the $\theta$ 's or $\epsilon^{\prime} s$.

If $\mathrm{x}_{\mathrm{n}}{ }^{\prime}$ is an agent's posterior variance over $\theta_{\mathrm{a}}$ at the end of the period, then from (9) the agent's posterior variance, $x_{n+1}$, over $\theta_{n+1}$, will be given by the function $h_{2}$ below of $x_{n}$ :

$$
h_{2}(x)=\alpha x+\sigma_{e}^{2}
$$

Hence that $x_{n+1}=h_{2}\left(x_{n}{ }^{\prime}\right)$. Suppose that at the beginning of the period an agent has a posterior variance over $\theta_{n}$ equal to $x_{n}$. Suppose that agent uses grade $n$. Then from (7) and (10) we 
Jovanovic and Nyarko The Transfer of Human Capical

conclude that the mapping from $x_{n}$ into $x_{n+1}$ is given by the mapping

$$
B(x)=h_{2}\left(h_{1}(x)\right)
$$

so that $x_{n+1}=B\left(x_{n}\right)$. If $m_{n}$ is the mean of the beliefs over $\theta_{0}$, then from (8) and (9), after $y_{a t}$ has been observed the posterior mean over $\theta_{0+1}$ will be

$$
m_{n+1}=\alpha^{1 / 2} h_{1}\left(x_{n}\right)\left\{m_{n} / x_{n}+y_{n} / \sigma_{w}^{2}\right\}
$$

It is easily verified that $B$ has a fixed point which we denote by $\bar{x}$. (See fig. 1). We assume that $\quad\{\alpha \mid<1$. The map on the right-hand side of eq. (10) then has a fixed point

$$
\hat{\mathrm{x}} \equiv \sigma_{\epsilon}^{2} /(1-\alpha)
$$

From $x$ iterates of the function $B$ converge monotonically to $\bar{x}$. (See fig. 1).

[Figure 1 about here]

We suppose that the ex ante distribution over $\theta_{0}$, the parameter of grade 0 , is its stationary distribution $-N(0, \hat{R})$. We assume that

$$
1-\hat{\mathrm{x}}-\sigma_{w}^{2}>0
$$

This means that the ex ante expected output on grade zero is positive.

2.3. Switching to a New Technological Line: We suppose that there is a continuum of technological lines available. Ex ante, each technological tine is the same. Each technological line has an associated sequence of grades $n=0,1,2, \ldots$. , and parameters $\left\{\theta_{n}\right\}_{n=1}^{\infty}$ as described in sections 2.1 


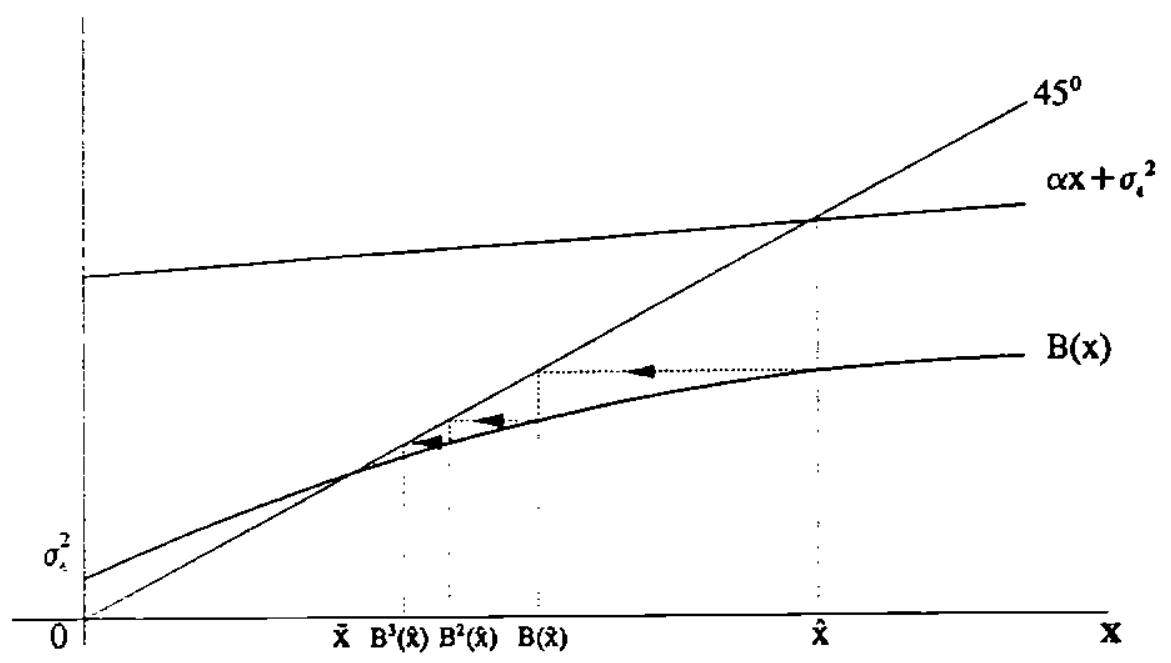

Figure 1: The Determination of $\hat{x}, \bar{x}$ and $B^{j}(x)$. 
Jovanovic and Nyarko The Transfer of Human Capieal

and 2.2. The $\left\{\theta_{n}\right\}_{n=1}^{\infty}$ sequence associated with one technological line is independent of the sequence associated with any other technological line. We impose the assumption

(A1) On an untried technological line, the ex ante belief over $\theta_{0}$ (and hence over $\theta_{a}$ for each $n=1,2,3, \ldots)$ is $N(0, \hat{x})$.

At date $\mathrm{t}$, any agent can choose on any technological line any grade $\mathrm{n}$ which is less than or equal to $t$. This means that the "production frontier" is moving out in step with time. (In Jovanovic and Nyarko (1994) this was called the Chari-Hopenhayn assumption.)

Suppose the agent is contemplating using a technological line over which he has no information. From (A1), his ex ante variance over $\theta_{n}$ is equal to $\hat{x}$ for all $n$. Then (14) implies that his expected output is the highest if he sets $n=t$. If an agent abandons a technological line whose grade is on the frontier, and starts a new technological line, the switch will represent a lateral switch - i.e., to the same grade $n=t$. (Jovanovic and Nyarko (1994) had only one technological line. Lateral switches were therefore not allowed in that paper.) In the model we present in the next section, some agents will indeed make lateral switches.

\section{The Overlapping Generations Structure}

We shall assume an overlapping generations structure with two-period lives. People are riskneutral, with lifetime preferences $c_{\text {young }}+\delta c_{\text {old }}$ where $c_{\text {young }}$ and $c_{\text {old }}$ denote the consumption when young and when old respectively, and where $\delta$ is the discount factor which we assume satisfies $0<\delta<$ $1 / \gamma$.

If the young and the old work together, the young can learn from the old. We shall refer to an old agent as "the foreman". A young agent working with a foreman will be referred to as "the apprentice." All decisions are made at the beginning of a period. Anything learned during the period can not affect decisions until the following period. 
Jovanovie and Nyarko The Transfer of Human Capital

We shall make the following assumption:

(A2) Agents are always on the production frontier (in the sense of $n=t$ ) of whatever technological line they are using.

We will provide justification for assumption (A2) in Appendix B.

3.1. The Foreman's Problem: First consider an old agent - a foreman - at some date t. Suppose that the foreman is using a technological line that has been in use since date 0 . By assumption the foreman will be at the frontier, and hence will be using a grade $n=t$. Therefore $n$ is also the age of the technology. Suppose that the belief of the foreman over the parameter $\theta_{n}$ is $N(m, x)$. The foreman will operate that grade according to (4) and will receive an expected payoff equal to $\gamma^{n}(1-$ $\left.\sigma_{w}{ }^{2}-x\right)$. The foreman at the beginning of the period also has to decide on whether or not to hire a young agent as an apprentice. If the foreman does not hire an apprentice he receives no further payoffs. He dies. So does that technological line!

Suppose on the other hand that the foreman does hire an apprentice. The apprentice will then use the technology of the foreman, and his grade, to produce output. As assumed in section 2.3, the apprentice, when hired, has a belief over $\theta_{\mathrm{a}}$ equal to its stationary distribution, $\mathrm{N}(0, \hat{\mathbf{x}})$. With this belief the apprentice chooses an action according to (4). In particular, he chooses the action $\mathrm{z}=0$. From (I) this results in an output of $I-y^{2}$. We suppose all of this output goes to the foreman. We now state the outputs and payments in units deflated by $y^{n}$. Using (3), the expected value of this output in terms of the expectations operator of the foreman, $E_{f}$, is given by:

$$
E_{1}\left(1-y^{2}\right)=1-\sigma_{w}^{2}-x-m^{2}
$$

In return for these labor services, the foreman pays the apprentice an amount $c_{n}$ (which we shall 
Jovanovic and Nyarke The Transfer of Humin Capital

characterize later). We refer to $c_{n}$ as the wage rate associated with labor on grade $n$. We may represent the value function and decision problem of the foreman by

$$
V_{n}(m, x) \equiv \operatorname{Max}\left\{1-x-\sigma_{w}^{2}, 1-x-\sigma_{w}^{2}+E_{r}\left(1-y^{2}\right)-c_{n}\right\}
$$

Equation (16) indicates that the decision of the firm is to hire or not hire. The definition in (16) is stated as if $\mathrm{n}$ and $\mathrm{x}$ are both free variables. We will later only study values of $\mathrm{x}$ which are some deterministic function of $n$, in which case we drop the $\mathrm{x}$ from the specification of the value function.

3.2. The Young Agent's Problem: The young may either be hired by a foreman or not hired. We first consider the case of an agent who is hired.

3.2a. If agent is hired: We now consider the situation of a young agent who is hired by a foreman using a grade $n$ of a technological line. The two, the young and the old that is, must then use the same grade. The apprentice will receive the amount $c_{n}$ in his first period as payment from the foreman. At the end of the period the young will receive information from two sources. The first of course is the observation of $y_{n}$ from use of that grade. The apprentice also learns by watching: he obtains information from observing the foreman's decision, $z$. The foreman sets $z$ to equal his own expectation of $\theta_{n}, E_{k} \theta_{n}$. Having seen his $z$ exactly, the apprentice learns the foreman's $m_{11}=E_{1}\left(\theta_{n}\right)$. Each fortman has a reputation in that it's widely known how much he knows, but not exactly what he knows. That is, the foreman's posterior variance over $\theta_{a}$ will be common knowledge. We shall denote this posterior variance by $x_{n}$. Since the foreman's beliefs are normally distributed, this implies that his beliefs are fully revealed once his $z$ is observed.

These two sources of information leaves the apprentice with beliefs over $\theta_{\mathrm{a}+1}$, the parameter of the next grade, which, from (9) is easily seen to be normal with mean and variance given by (12) and (11). In the next period the young agent becomes old. He becomes a foreman and solves 
Jovanovic and Vyarko The Transfer of Human Capital

the foreman-problem described earlier, but with grade $n+1$. His lifetime return (deflated by $\gamma^{n}$ ) is therefore

$$
c_{n}+\delta \gamma E_{a}\left[V_{n+1}\left(m_{n+1}, x_{n+1}\right)\right]
$$

where $E_{\mathrm{j}}$ denotes his expectations operator over $m_{\mathrm{n}+i}$ when he is young. We shall define $E_{\mathrm{n}}$ precisely in equation (30).

We assume that it takes an entire period for the young to observe the action choice of the old. In particular, the old can not merely i form the young of his beliefs at the beginning of the period betore production takes place. We envisage a situation where the young actually have to watch the old. and that this takes an entire period. Each apprentice therefore chooses an action $z=0$. The foreman with beliefs which are normal with mean $m$ however believes that the optimal decision is $\mathrm{z}=\mathrm{m}$. Hence that forenan believes that the young will make "mistakes." When $\mathrm{m}$ is large those mistakes are large. Hence not every foreman will take on an apprentice. Foremen will have skills specific to technologies on which young agents are highly unproductive. This would be a technology on which $\left|\theta_{11}\right|$ has revealed itself (to the old agent) to be large. If hired, a young agent make costly mistakes. Such technologies will therefore die with the foreman who operates them, and new technologies will be started by young agents that did not join up with a foreman.

3.2b. "Going it Alone:" Suppose that the young agent is not hired by the foreman who was about to use grade $n$ of a technological line. The young agent then starts a new technological line. From (A2) the young agent uses grade $n$ of the new technological line. From (A1), the young agent's belief over the parameter of grade $n$ of the new technological line is $N(0, \hat{x})$. This is of course the same as the ex ante distribution of $\theta_{0}$ of the original technological line. Indeed, after deflating by $\gamma^{n}$, the expected output of this agent on the new technological line is equal to the expected output on grade 0 of the original technological line. This deflated output is equal to $1-\hat{x}-\sigma_{w}{ }^{2}$. Let $y_{0}$ denote his observation from the use of that grade. Then from (12) and (11) his posterior belief over the 
Sovanovic and Nyarke The Transfer of Human Capial

next grade after observing $y_{0}$ is $N\left(m_{1}, x_{1}\right)$ where

$$
m_{1}=\alpha^{1 / 2} h_{1}(\hat{x}) y_{0} / \sigma_{w}^{2} \quad \text { and } \quad x_{1}=B(\hat{x})
$$

His expected lifetime utility, discounted by $\gamma^{n}$, is therefore given by

$$
u_{0} \equiv:-\hat{x}-\sigma_{w}{ }^{2}+\delta \gamma E_{a} v_{1}\left(m_{1}, x_{1}\right)
$$

where $E_{2}$ denotes expectations over the random variable $m_{1}$ defined in (18). $u_{0}$ is the value to the young of "going it alone" - when not hired by a foreman. This is in units discounted by $\gamma^{\text {n }}$. Implicit in (19) is a stationarity assumption which says that calendar time matters only in as much as it affects production possibilities. We say more about this in section 5 .

3.2c. Young agents are perfect substitutes. Old agents are not; they have learned a specific skill. In particular they have information on a technological line. For now we assume that all the rents from the partnership go to the old. Later on we show that some young agents are not hired in equilibrium. Competition then results in the old receiving all the rents. This implies that in equilibrium the value to the young from being hired, i.e., (17), will equal the return from going it alone, (19). This implies that

$$
c_{n}=1-\hat{x}-\sigma_{w}{ }^{2}+\delta \gamma\left\{E V_{1}\left(m_{1}, x_{1}\right)-E_{2}\left[V_{n+1}\left(m_{n+1}, B\left(x_{n}\right)\right)\right]\right\}
$$

Putting this value of $c_{n}$ into the foreman's problem (16) and simplifying implies that

$$
V_{n}(m, x)=1-x-\sigma_{w}{ }^{2}+\operatorname{Max}\left\{0, g_{n}-m^{2}\right\}
$$

where $g_{n}$ is independent of $m$ and is given by 
Jovanovic and Nyarko The Transfer of Human Capital

$$
\left.\left.g_{n}=\hat{x}-x_{n}+\delta \gamma\left[E_{2} V_{n+1}\left(m^{\prime}, B\left(x_{n}\right)\right)\right]-E_{2} V_{1}\left(m_{l}, B(\hat{x})\right)\right]\right\}
$$

Suppose that the foreman is of type $(m, x)$ with $m=0$. Then that foreman would himself set $z=0$ when operating his technology. A young agent using his technology always sets $m=0$. The expression $g_{n}$ above is the net output such a foreman receives from the young, $E\left(1-y^{2}\right)-c_{n}$. If the old is of type $(m, x)$ with $m \neq 0$, that old person would prefer the young to choose the decision $z=m$. However, the young always choose action $z=0$. As far as the old is concerned, the young would be making "Inistakes" when operating his technology. The total net output that the old receive from the young if hired, is $\mathrm{g}_{n}-\mathrm{m}^{2}$. The amount $-\mathrm{m}^{2}$ therefore represents the losses or costs due to the mistakes of the young. If these are large the old will then decide not to hire the agent.

$A$ foreman of type $(m, x)$ will therefore hire a young agent at date $n$ if $g_{n}-m^{2}>0$ (and will be indifferent between hiring and not hiring if equality holds). We define

$$
\begin{aligned}
& \overline{\mathrm{m}}_{\mathrm{n}}(\mathrm{x}) \equiv\left(\mathrm{g}_{\mathrm{n}}\right)^{1 / 2} \text { if } \mathrm{g}_{\mathrm{n}}>0, \quad \text { and } \\
& \overrightarrow{\mathrm{m}}_{\mathrm{n}}(\mathrm{x}) \equiv 0 \text { othervise. }
\end{aligned}
$$

A foreman of type $(m, x)$ will hire a young agent whenever $m$ lies in the set $A_{n}(x) \equiv\left[-\vec{m}_{n}(x), \bar{m}_{n}(x)\right]$. We call $\bar{m}_{n}(x)$ a hiring rule, and $A_{n}(x)$ a hiring set. A foreman is more likely to hire a young agent the smaller is the foreman's $|\mathrm{m}|$.

The $A(x)$ correspondence defines the hiring set in $(m, x)$ space, and it is shown as the shaded area in figure 2. Recall that $\bar{x}$ is the fixed point of the operator $B$. Since $B(\hat{x})<\hat{x}$, and $B(x)$ is as shown in figure 1 , the sequence $B^{j}(\hat{x})$ declines monotonically from $\hat{x}$ to $\bar{x}$ as shown in this figure. This is why in figure 2, values of $\mathbf{x}$ below $\bar{x}$ or above $\hat{\mathbf{x}}$ could not arise.

[figure 2 about here].

At the date 0 we suppose that the old at that date have the same beliefs about the technology as do the young. In particular, $m_{0}=0$ and the variance of $m_{0}$ is zero. The young receive no extra 


\section{Jovanovic and Nyorko The Transfer of Human Capital}

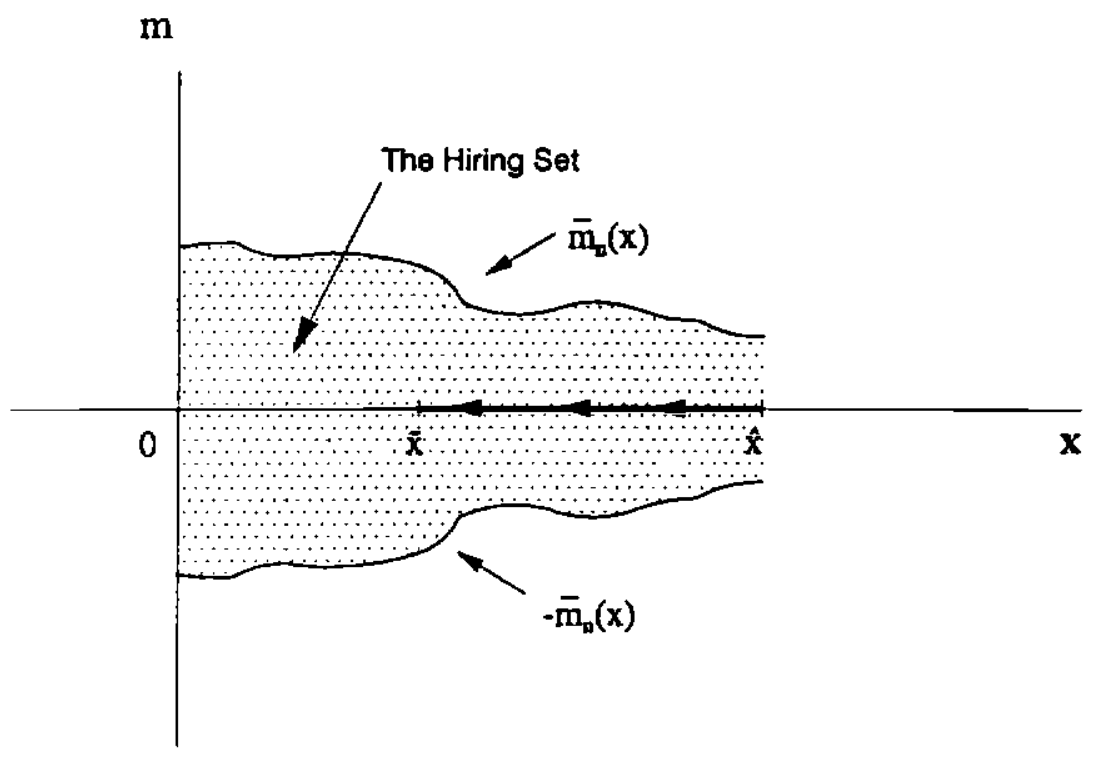

Figure 2: The Hiring Set. 
Jovanovic and Nyarko The Transfer of Human Capinal

information after using the technology of the old at that date as opposed to going it alone. If indeed the young were hired they would be paid an amount when young, $c_{p}$, equal to what they would receive trom going it alone $1-\sigma_{w}{ }^{2}-\hat{\mathbf{x}}$.

3.3. The Transfer of Information Along a Technological Line First consider the following slight digression. Suppose two agents $\mathrm{A}$ and $\mathrm{B}$ have the same prior over some parameter $\theta$. Suppose $\mathrm{A}$ receives some information and forms a posterior over $\theta$. Suppose that $B$ does not receive that information but observes the posterior of agent $A$. What then is B's posterior over $\theta$ ? It will of course be equal to the posterior of $A$. Despite not knowing precisely the information of $A, B$ says to himself "any information that A could have seen would lead me to have a posterior equal to the announced posterior of $A$ since I have the same prior as A. Hence, despite the fact that I did not see $A$ 's information, my posterior is the same as that of A."

The agent who is young at date 0 has a prior $N(0, \hat{\mathbf{x}})$ over $\theta_{0}$. That agent then observes $y_{0}$ and uses this to form a posterior over $\theta_{0}$, and hence over $\theta_{1}$. His posterior over $\theta_{1}$ is then $N\left(m_{1}, x_{1}\right)$. Suppose he hires at date 1 (or more precisely the first period in the life of the technological line) and announces $m_{1}$ to the young at date 1 . The young at that date do not see $y_{1}$; only $m_{1}$. What will be that agent's belief over $\theta_{1}$ ? From the argument made earlier, the young at date 1 will have the same posterior, $N\left(m_{1}, x_{1}\right)$, over $\theta_{1}$ as the old at date 1 . In particular, observation of $m_{1}$ is equivalent to observation of $y_{0}$ as regards forming a posterior over $\theta_{1}$. We may extend this argument inductively to each period $n$. In particular, consider the young who observe $m_{v}$, the posterior mean over $\theta_{n}$ of the old. Conditional on observing $r_{\text {in }}$, the young will have a posterior over $\theta_{\text {, which }}$ is $N\left(m_{0}, x_{n}\right)$. In particular, the young will inherit the beliefs of the old. Furthermore, the belief of the young conditional on observing $m_{b}$ is the same as the beliefs that the young would have if they had observed all of the signals $Y_{n-1} \equiv\left\{y_{0} y_{1}, \ldots, y_{n-1}\right\}$ that all agents of earlier periods had observed on that technological line. $m_{n}$ is a sufficient statistic for the entire information vector $Y_{n-1}$.

A foreman using a grade $n$ will therefore have a posterior variance over the parameter $\theta_{n}$ 
Jovanovic and Nyarko. The Transfer of Human Capital

equal to $x_{n}=B^{n}(\hat{x})$. The value function of the foreman is therefore a function only of his prior mean $m_{n}$ and the age of the technology. We define

$$
V_{n}(m) \equiv V_{n}\left(m, B^{n}(\hat{x})\right), \quad \bar{m}_{n} \equiv \bar{m}_{n}\left(B^{n}(\hat{x})\right), \quad \text { and } \quad A_{n} \equiv A_{n}\left(B^{n}(\hat{x})\right)
$$

and use this new notation throughout.

\section{The $\left\{m_{n}\right\}^{n}=1$ Process}

4.1. Suppose that the posterior belief over $\theta_{n}$ is $N\left(m_{n}, x_{n}\right)$. From (12) and (3), after the signal $y_{n}$ has been observed $m_{n+1}$, the posterior mean over $\theta_{n+1}$, is given by

$$
m_{n+1}=\alpha^{1 / 2} h_{1}\left(x_{n}\right)\left\{m_{n} / x_{n}+\left(\left(\theta_{n}+w_{n}\right) / a_{w}{ }^{2}\right\}\right.
$$

Conditional on $m_{n}$, the posterior over $\theta_{n}$ is $N\left(m_{n}, x_{n}\right)$. Hence conditional on $m_{n}, m_{n+1}$ is normal with mean and variance given below:

$$
\begin{aligned}
& E\left[m_{n+1} \mid m_{n}\right]=\alpha^{1 / 2} h_{1}\left(x_{n}\right) m_{n}\left\{1 / x_{n}+1 / o_{w}{ }^{2}\right\}=\alpha^{1 / 2} m_{n} ; \\
& \operatorname{Var}\left[m_{n+1} \mid m_{n}\right]=\left[\alpha^{1 / 2} h_{1}\left(x_{n}\right)\right]^{2}\left(1 / \sigma_{w}{ }^{2}\right)^{2}\left[x_{n}+\sigma_{w}{ }^{2}\right]=\alpha h_{1}\left(x_{n}\right)\left(1 / o_{w}{ }^{2}\right) x_{n 1} ; \text { and } \\
& E\left[m^{2}{ }_{n+1} \mid m_{n}\right]=\alpha h_{1}\left(x_{n}\right)\left(1 / \sigma_{w}{ }^{2} x_{n}+\alpha m_{n}^{2} .\right.
\end{aligned}
$$

Equations (26) and (27) completely describe the distribution of $m_{p+1}$ conditional on $m_{p}$, which we denote by Prob $\left(. \mid m_{n}\right)$. The unconditional variance of $m_{n}$ is given by the recursion

$$
\operatorname{Var} m_{n+1}=\alpha h_{1}\left(x_{n}\right)\left(1 / \sigma_{w}{ }^{2}\right) x_{n}+\alpha \operatorname{Var} m_{n} \quad \text { and } \quad \operatorname{Var} m_{0}=0
$$


Jovanovic and Nyarko The Transier of Humen Capital

We detine

$$
E_{a}=E\left[. \mid A_{1}, \ldots, A_{n}\right]
$$

to be the expectation operator over the $\left\{m_{n}\right\}_{n}$ process conditional on some hiring sets $A_{1}, \ldots, A_{n}$, where $A_{r}=\left[-\bar{m}_{r}, \bar{m}_{r}\right]$ for some $\bar{m}_{t}>0$. (This operator was introduced in (17).)

We now have the following:

Proposition 4.1: Fix any $n$.

(a) Prob $\left[m_{n-1}{ }^{2} \leq m_{0} \mid m_{n}=m\right]$ decreases as $|m|$ increases.

(b) For each $n$, Prob $\left[m_{n+1}{ }^{2} \leq m_{0} \mid m_{n} \leq m\right]$ decreases as $|m|$ increases.

(c) The distribution of $m_{n+1}{ }^{2}$ (resp. $\left.\left|m_{n+1}\right|\right)$ under the probability defining the conditional expectations $E\left[. m_{n}=\bar{m}_{11}\right]$ first order stochastically dominates distribution of $m_{n+1}^{2} \quad$ (resp. $\left.\left|m_{n-1}\right| \mid\right)$ under the probability defining $E_{a}$ in (30) where $A_{r}=\left[-\bar{m}_{r}, \bar{m}_{r}\right]$.

Corollary 4.2.: The distribution of any increasing monotone transform of $\mathbf{m}_{n+1}^{2}$ (e.g., $\left.\left|\mathrm{m}_{n+1}\right|\right)$ is also similarly stochastically increasing in $\left|m_{n}\right|$. In particular all statements in Proposition 1 also hold when $\mathrm{m}_{n+1}^{2}$ is replaced with an increasing monotone transform of $\mathrm{m}_{n+1}^{2}$.

Remark: Fix $m_{0}$ and $m$. Prob $\left(m_{n+1}^{2} \leq m_{0} \mid m_{n}=m\right)$ can not be similarly ordered in terms of $n$.

4.2. The young Agent's Ex ante belief about $m_{n}$. Consider a young agent who is considering whether or not to work with a foreman who has a technology of vintage $n$. What will be that agent's belief about the value of the posterior mean of the old agent, $m_{n}$. Note that this belief and 
Jovanovic and Nyarko The Transfer of Human Capial

(12) determines the young agents expectation operator $E_{2}$ over $m_{n+1}$ which is what was required in (17) to state the return to the young from working with the old.

The young will not use the unconditional distribuition, determined by (26) and (27) above to form expectations over $m_{11}$. The young agent knows the age, $n$, of the technology. This implies that the foremen in previous periods all hired young agents. This in turn implies that the $m_{1}$ process fell in the hiring set $A_{1}$ in each of the previous periods of the life of that technological line. The young agent will condition on this information in forming a beliefs about the value of $m_{0}$.

Fix a sequence of hiring sets $A_{n}=\left[-\bar{m}_{n}, \bar{m}_{n}\right]$. In particular, an old agent using a techology of age $n$ will hire a young agent if and only if his mean $m_{n}$ lies in the set $A_{n}$. Let $\sigma_{a}{ }^{2}$ be the variance of $m_{u}$ conditional on knowledge that $m_{r}$ was in $A_{r}$ for $r=0, \ldots, n-l$. This is the belief of an agent whose only information is that the technological line has been alive in periods $r=0,1, \ldots, n-1$. Then we have

Proposition 4.3: $a_{n \cdot 1}^{2}$ obeys the pair of inequalities

$$
\frac{\alpha h_{1}\left(x_{0}\right) x_{n}}{\sigma_{w}^{2}} \leq \sigma_{n \cdot 1}^{2} \leq \frac{\alpha h_{1}\left(x_{n}\right) x_{n}}{\sigma_{w}^{2}}+\alpha \sigma_{n}^{2},
$$

with $\sigma_{0}^{2}=0$.

5. Existence and Pronertics of Equilibrium: We now define an equilibrium for the model. Our definition will implicitly require that some amount of hiring takes place on each grade $n$, at least for the foreman with $m_{n}=0$. We show in appendix $A$ that any equilibrium must be of this kind. 
Jowenovic and Vyarko The Transfer of Human Capila!

Definition: An equilibrium is a sequence of wage rates $\left\{c_{n}\right\}_{n \rightarrow \infty}^{\infty}$ such that if we define

$g_{n} \equiv 1-x_{n}-\sigma_{w}{ }^{2}-c_{n} ; \quad A_{n} \equiv\left\{m m_{n}^{2} \leq g_{n}\right\} ; V_{n}(m) \equiv 1-x_{n}-\sigma_{w}{ }^{2}+M a x\left\{0, g_{n}-m^{2}\right\} ; \quad$ and $u_{0} \equiv 1-\hat{x}-\sigma_{w}{ }^{2}+E V_{1}\left(m_{1}\right)$

then for each $n=1,2, \ldots .$,

$$
\begin{aligned}
& \left.u_{0}=c_{n}+\delta \gamma E\left[V_{n+1}\left(m_{n+1}\right)\right] A_{1}, \ldots, A_{n}\right] \text { (no excess supply of young labor); } \\
& 1-\sigma_{w}^{2}-x_{n}-c_{n} \geq 0 \text { (Hiring occurs on grade } n \text { for some } m_{n} \text { values). }
\end{aligned}
$$

In the above $\left\{m_{n}\right\}_{n=1}^{x}$ is the stochastic process of means described in section 4 .

It is easy to show that in any equilibrium the value functions $\left\{V_{n}\right\}_{n=1}^{\infty}$ obey the functional equation in (21) and (22). Given an equilibrium, using the notation in the definition above we see that the hiring rule is given by $\bar{m}_{n} \equiv g_{n}{ }^{1,2}$, and the hiring set is given by $A_{n}$, for each $n$.

In appendix $A$ we show that equilibria exist. Not only that, there are many equilibria. The equilibria are indexed by the value of the age one value function at $m=0, v_{1}(0)$. In particular, for each real number $v$ greater than or equal to some critical value, which we refer to as $\bar{v}$, there exists an equilibrium with $V_{1}(0)=v$. (See remark $R I$ of Appendix A.)

The unique equilibrium such that $V_{1}(0)=\bar{v}$ will be referred to as the bounded equilibrium. (See Remark R2 of appendix A.) '?: bounded equilibrium has the following interpretation: . Let us consider a finite horizon equilibrium where we insist that the decision problem is the same as our earlier description for all agents with technology of age $n \leq N+1$, but where we insist that no hiring is allowed on technologies of age $\mathrm{N}+1$ or larger. This means that we "kill" all technologies precisely when they get to age $N+2$, regardless of whether or not young agents would want to work on such technologies. It turns out that there exists a unique finite horizon equilibrium of this sort. The unique bounded equilibrium is the limit of the finite horizon equilibria, as the horizon, $\mathrm{N}$, goes to infinity.

Each equilibrium with $V_{1}(0)$ strictly greater than $\bar{v}$ will be such that 
Jomanovic and Nyanko The Transfer of Human Capieal

$$
\lim _{n \rightarrow \infty} V_{n}(m)=\infty \text { for all } m \text {. }
$$

We refer to such equilibria as Ponzi equilibria.

One thing that comes out of our construction of equilibria is:

Proposition 5.1.: In any equilibrium, the hiring rule, $\vec{m}_{n}$, is strictly positive for each $n=1,2, \ldots$; and the hiring set $A_{n}=\left\{-\bar{m}_{n}, \bar{m}_{n}\right]$ is a non-empty non-degenerate interval for each $n$.

Corollary 5.2: In any equilibrium, for each $\mathrm{N}<\infty$, there exists a strictly positive probability that the length of life of the technological line will exceed $N$.

Below. we show that in any Ponzi equilibrium $c_{n}$, the wage payment of the old to the young for their labor tends to minus infinity so will eventually be negative. This means that the young pay the old. This is in return for the information that the old will transfer to the young.

Proposition 5.3. In any Ponzi equilibrium $\lim _{a \rightarrow \infty} c_{n}=-\infty$.

It should be clear what is going on in the Ponzi equilibria. Suppose that at date 0 a young agent starts a technological line. Any arbitrarily high value for the date one value function, $\mathrm{V}_{\mathrm{l}}$, may be obtained by setting $c_{1}$ equal to some large negative number. The date one young are willing to pay this amount because they expect that when they get old the young then will pay them back an even higher amount. That even higher amount is justified by yet higher payments. Etc. Etc.

Regardless of whether we are in a bounded or Ponzi equilibrium, the lifetime payoff to any agent is equal to $u_{0} \equiv I-\hat{x}-\sigma_{w}{ }^{2}+\delta \gamma E_{1}\left(m_{1}\right)$. Hence despite the fact that along a given Ponzi equilibrium $V_{n}(m) \rightarrow \infty$, each agent receives the same finite discounted payoff of $u_{0}$. The reason for this is simple. Even though $V_{n}(m) \rightarrow \infty$, so too does $c_{n} \rightarrow-\infty$. The payoff to the agent who is young at $n$ is $c_{n}+\delta \gamma E V_{n+1}\left(m_{n+1}\right)=u_{0}<\infty$. Implicit in all of the paper and indeed in the 
Jovanovic and Nyarto The Transfer of Human Capipl

construction of equilibria above is the following: We rule out equilibria of a particular type. We do not consider equilibria where the age-one value function, $V_{1}(m)$, depends upon the date. Suppose that from dates $I$ through $T$ technological line $A$ is in used. At date $T$ no young are hired on that line and it dies. The young form a new technological line $B$ at that date. Let $V_{1}{ }^{A}$ and $V_{1}^{B}$ denote the age-one value function corresponding to technological lines A and B. Since we have multiple equilibria it is in principle possible that at date $T$ the young form a technological line with a different associated Ponzi equilibrium than that of line $A$. In that case $V_{1}{ }^{A}+V_{1}^{B}$. This is a form of timenonstationarity. If we allowed for this inequality we would then have to index our value functions by not only the age of the technology, but also by the calender time. We rule out such time nonstationarities. Note however that this does not imply in our model that $\mathrm{V}_{\mathrm{n}}=\mathrm{V}_{\mathrm{n}+1}$.

\section{The Planner's Problem}

6.1. The planner wishes to maximize the discounted sum of utilities of all the generations starting at date zero. This means he will maximize discounted aggregate output. As in the case of equilibrium, the planner's problem can be recast in a stationary form, with a discount factor of $\gamma \delta$.

The only difference between the Planner's problem and the equilibrium solution is in the hiring sets. The planner chooses the hiring set to maximize aggregate output of all generations. The equilibrium hiring set is chosen to maximize the payoff of the foreman doing the hiring. In this section we shall compare the hiring sets in the two situations.

In the equilibrium solution, the discounted aggregate output may be computed by adding the output of each foreman to that of the lifetime output of the current and future young. The young at any future date will have an expected output equal to $u_{0}$ given in (19). The equilibrium utility of an old agent with technology of grade $n$ and beliefs represented by $(m, x)$ was denoted by $V(m, x)$. The equilibrium lifetime utility of the young agent is $u_{0}$. Hence the equilibrium utility of the foreman of type $(m, x)$ plus the discounted lifetime utility of the young agent that he interviews, plus the discounted output of the agent that this young agent interviews in the subsequent period, and 
Jovonovic and Vyarko The Transfer ol Human Capikd

so on into the infinite future, is equal to

$$
W_{n}{ }^{e}(m) \equiv V_{n}(m)+u_{0}(1-\delta \gamma)
$$

The value of $W_{n}^{e}$ does not represent the maximum possible discounted output stream. The planner will wish to follow a hiring policy that will, as a function of $\mathrm{m}$ and $\mathrm{x}$, maximize the analog of $W_{11}:$

The planner has the same information as the foreman does; that is, he knows $m$ and $x$. If a forcman of type ( $m, x)$ hires a young agent, the planner's expectation of their joint output is

$$
\left[1-x-\sigma_{w}^{2}\right]+\left[1-m^{2}-x-\sigma_{w}^{2}\right]=2\left(1-x-\sigma_{w}{ }^{2}\right)-m^{2}
$$

If the young agent is to work alone, the planner must assign him a random new technological line, and so he expects the joint output of the two agents to be

$$
\left[1-x-\sigma_{w}^{2}\right]+\left[1-\hat{x}-\sigma_{w}^{2}\right]
$$

Let $W_{11}(m)$ be the planner's expectation of the infinite sequence of such pairs of outputs. It solves the equation

$$
\begin{aligned}
W_{n}(m)=\operatorname{Max}\left\{2\left(1-x_{n}-\sigma_{w}{ }^{2}\right)-m^{2}\right. & +\delta \gamma E\left[W_{n+1}\left(m_{n+1}\right) \mid m_{n}=m\right], \\
& \left.1-x_{n}-\sigma_{w}{ }^{2}+1-\hat{x}-\sigma_{w}{ }^{2}+\delta \gamma E W_{1}\left(m_{1}\right)\right\}
\end{aligned}
$$

where the first expectation is over $m_{n+1}$ given $m_{n}$ (defined via (26) and (27)) and the second is over $m_{1}$ defined in (18). Using standard contraction mapping techniques it should be easy to see that there exists a unique sequence of continuous functions $\left\{W_{n}\right\}_{n-1}^{\infty}$ satisfying (36). 
Lemma 6.1: $W_{n}(m)$ is decreasing in $|m|$ for each fixed $n$.

Since the top line of (36) is a decreasing function of $\left|\mathrm{m}_{\mathrm{n}}\right|$, or equivalently, of $\mathrm{m}^{2}$, while the second line of (36) does not depend on $m_{n}$, the planner will choose a cutoff value for $\left|m_{n}\right|$, call it $m_{n}^{u p t}$ and reject all matches for which $\left|m_{n}\right|$ exceeds this value:

$$
m_{n}^{\text {opl }}=\left\{\hat{x}-x_{n}+\delta \gamma\left\{E\left[W\left(m_{n+1}\right) \mid m_{n}^{o p}\right]-E W_{1}\left(m_{1}\right)\right\}\right\}^{1 / 2} .
$$

The equilibrium hiring set cutoff, $\bar{m}_{n}$, is given by (23):

$$
\bar{m}_{n}=\left\{\hat{x}-x_{n}+\delta \gamma\left\{E_{a}\left[V_{n+1}\left(m_{n+1}\right)\right]-E_{a} V_{1}\left(m_{1}\right)\right\}\right\}^{1 / 2}
$$

From (33) this becomes

$$
\bar{m}_{n}=\left\{\hat{x}-x_{n}+\delta \gamma\left\{E_{a}\left[W_{n+1}^{c}\left(m_{n+1}\right)\right]-E_{2} W_{1}{ }^{c}\left(m_{1}\right)\right\}\right\}^{1 / 2}
$$

Since $W_{n}$ is the outcome of socially optimal decisions,

$$
W_{n}(m) \geq W_{n}{ }^{c}(m) \text { for all } m \text {. }
$$

Recall that $E_{a}$ in (38) represents expectations over $m_{n+1}$ conditional only on information that the $m_{r}$ iell in the hiring set, $A_{r}$, in each of the previous periods in the life of the current technology line. Consider a planner at some fixed date $t$ observing the equilibrium in process. At that date the old will be using a technology of age $n$. Suppose that the planner knows the value of $m_{a}$ of 
Jovanovic and Nyarko The Transfer of Human Capital

the old agent using that technology. If the planner had the opportunity to change the hiring decision at that date only, would the planner choose to increase or to decrease the hiring set? It turns out that the planner would want to decrease it!

Let $m_{1}^{*}$ be the optimal hiring rule under the conditions just mentioned. The planner 's value function is then given by the relation analogous to (36):

$$
\begin{aligned}
W_{n}(m)=\operatorname{Max}\left\{2\left(1-x_{n}-\sigma_{w}{ }^{2}\right)-m^{2}\right. & +\delta \gamma E\left[W_{n+1}^{e}\left(m_{n+1}\right) \mid m\right], \\
& \left.1-x_{n}-\sigma_{w}{ }^{2}+1-\hat{x}-\sigma_{w}{ }^{2}+\delta \gamma E W_{1}\left(m_{1}\right)\right\}
\end{aligned}
$$

Eq. (41) emphasizes the problem of the planner in our experiment: The planner is deciding the decision rule for a technology of age $n$ at some fixed date $t$. Given $m$ the planner must decide whether or not to allow a young to be matched with a foreman with mean $\mathrm{m}$, or whether to inform the young to go it alone. If the young is hired at date $t$, then in each subsequent date the equilibrium is played out. Hence we see the equilibrium value function $\mathrm{W}_{\mathrm{n}+1}{ }^{e}$ on the top line of (41). Suppose on the other hand the planner recommends that no young are hired by the foreman of type $m$. Then the young goes it alone. If we suppose that young agents re-start the equilibrium outcome, then $W_{1}{ }^{*}$ on the second line of $(41)$ is the equilibrium infinite horizon value function; i.e. $W_{1}{ }^{*}=W_{1}{ }^{e}$. If alternatively the young star:-up the planner's problem, then $W_{1}^{*}=W_{1}$. From (40) we therefore have that in either case

$$
W_{1}^{*}(m) \leq W_{1}(m) \text { for all } m
$$

Using Corollary 4.2 in addition to arguments similar to that of Lemma 6.1, it is easy to see that the top line of (41) is decreasing in $|\mathrm{m}|$. The second line in (41) is independent of $\mathrm{m}$. Hence the solution to the problem in (41) and (42) is characterized by a hiring rule $m_{a}^{*} \geq 0$. The planner will 
Jovanovic and Nyarko The Transfer of Human Capital

recommend that the young be hired whenever $m_{\mathrm{p}}$ lies in $\left[-\mathrm{m}_{\mathrm{n}}^{*}, \mathrm{~m}_{\mathrm{p}}^{*}\right]$, and will recommend no hiring when $m_{n}$ lies outside of this interval.

We now have the following:

Proposition 6.2: Suppose that the planner may make a one time change in the hiring rule. In particular suppose that the planner is solving the problem characterized by (41) and (42) for some fixed $\mathrm{n}$ at some date $\mathrm{t}$ Then the planner will choose to reduce the hiring set; i.e., $m_{\mathrm{n}}^{*} \leq \bar{m}_{\mathrm{n}}$. The inequality is strict whenever $\bar{m}_{n}>0$. This means that if there is ever a non-trivial amount of hiring, that amount hiring is excessive.

[Figure 3 about here].

Proposition 6.2 is true because the informational asymmetry between foremen and young agents gives rise to an adverse selection problem. The marginal technology, $\bar{m}_{n}$, is the worst among continuing technologies. When he hires, the marginal foreman ignores the fact that he is contaminating the distribution of surviving technologies. Therefore, in equilibrium, there is a tendency to stick too much to old methods and the startup rate of new technologies is too low.

6.2. Implementing the ontimal policy. The planner can implement the optimal limited intervention policy without knowing any of the $\mathrm{m}$ 's. If he chooses to intervene at the point at which technologies are $n$ periods old, equation (38) implies that he can induce old agents to be indifferent between hiring at $m=m_{n}$ by imposing a hiring tax $\tau_{n}$ given by

$$
\tau_{n}=\left(\vec{m}_{n}\right)^{2}-\left(m_{n}^{*}\right)^{2}
$$


Jovanovic and Nyarko The Transfer of Human Capilal

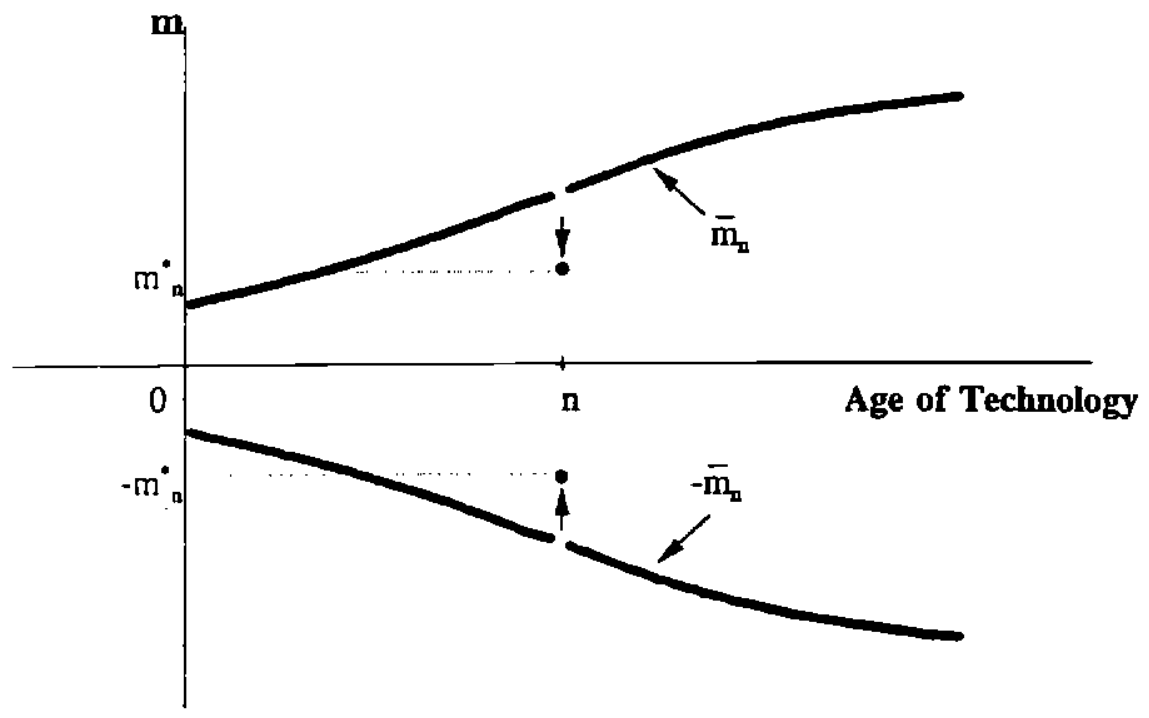

Figure 3: The Optimal Limited Intervention. 
Jovanovic and Nyarko The Transfer of Human Capital

7. Overlapping Generations Learning Versus Frictionless Learning. How well does the OG mechanism approximate learning by, say, an infinitely-lived individual who sees the same evidence as the old and young agent combined? Since $h_{1}(x)=x \sigma_{w}^{2} /\left(x, \sigma_{w}^{2}\right)$, proposition 4.3 implies

$$
\frac{\alpha x_{n}^{2}}{x_{n} \cdot \sigma_{w}^{2}} \leq \sigma_{n-1}^{2} \leq \frac{\alpha x_{n}^{2}}{x_{n}+\sigma_{w}^{2}}+\alpha \sigma_{n}^{2} .
$$

We call this the overlapping generations (OG) case.

Alternatively, suppose that an infinitely lived individual sees the signals of both the young and old agents in each period. In this case, the difference equation for $\sigma_{q}^{2}$ becomes

$$
\sigma_{n \cdot 1}^{2}=\frac{\alpha x_{n}^{2}}{2 x_{n}+\sigma_{w}^{2}}+\alpha \sigma_{n}^{2},
$$

since there are now two signals per period, and there is no truncation (i.e., $\bar{m}=\infty$ ). We call this the frictionless case.

The situation is shown in figure 4. The top two lines are straight, with slope $\alpha$. The bottom line has slope zero. As $x_{n}$ declines, all three lines in figure 4 shift down, but if all are evaluated at the same $x_{n}$, their relative positions remain unchanged. Equation (44) implies that for $\sigma_{n}^{2}$ given, $o_{n+1}^{2}$ must, in the $O G$ case, lie in the shaded area. So from any fixed prior variance $x_{p}$, the frictionless case involves faster learning, which is reflected in the greater height of its curve, and a more rapid growth in $o_{n}{ }^{2}$. The least learning (none!) occurs for the case $\alpha=0$, in which case $\mathrm{O}^{2}{ }_{n+1}=0$ for all $\mathrm{n}$, for both the $\mathrm{OG}$ and the frictionless cases.

[Figure 4 about here]. 


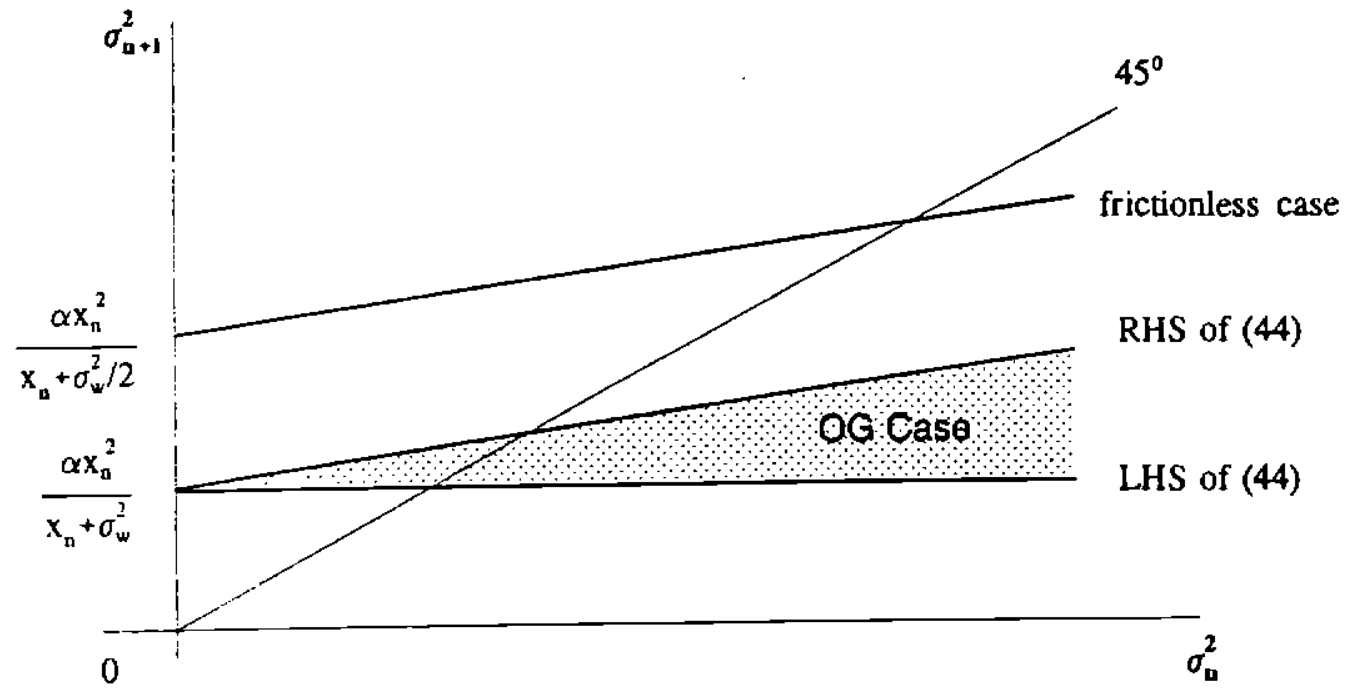

Figure 4: The $\sigma_{0}^{2}$ process in the $O G$ and the frictionless cases. 
Jovanovic and Vyarko The Transfer of Human Capital

The faster learning in the frictionless case also implies a lower $x_{n}$ sequence. From equation (10). the OG case implies the following difference equation for $x_{n}$ on a surviving technology:

$$
x_{n-1}=\frac{\alpha x_{n}{ }^{2}}{x_{n} \cdot \sigma_{w}^{2}} \cdot \sigma_{c}^{2},
$$

whereas in the frictionless case it is

$$
x_{n \cdot 1}=\frac{\alpha x_{n}^{2}}{2 x_{n}+\sigma_{w}^{2}}+\sigma_{c}^{2} .
$$

These two curves are drawn in figure 5; the top one pertains to the OG case. Taking as the initial condition for both cases implies that at each date $x_{n}$ is higher for the OG case, and it must also have a higher limit, denoted by $\tilde{x}_{\mathrm{OG}}$ as opposed to $\tilde{\mathrm{x}}_{\mathrm{F}}$ for the frictionless case. Output and productivity are, at each date, both lower in the OG case, not just at each date, but in the limit as well.

[Figure 5 around here].

If there were no upgrading, the productivity differences between the $O G$ and the frictionless case would disappear in the long run, because even OG learning would eventually perfectly reveal the parameter(s) of interest. But because of continual upgrading, ignorance about $\theta_{n}$ is always there, but it is permanently larger in the OG case. In the limit, both economies grow at the rate $\gamma-1$, but the differences in productivity levels remain. 


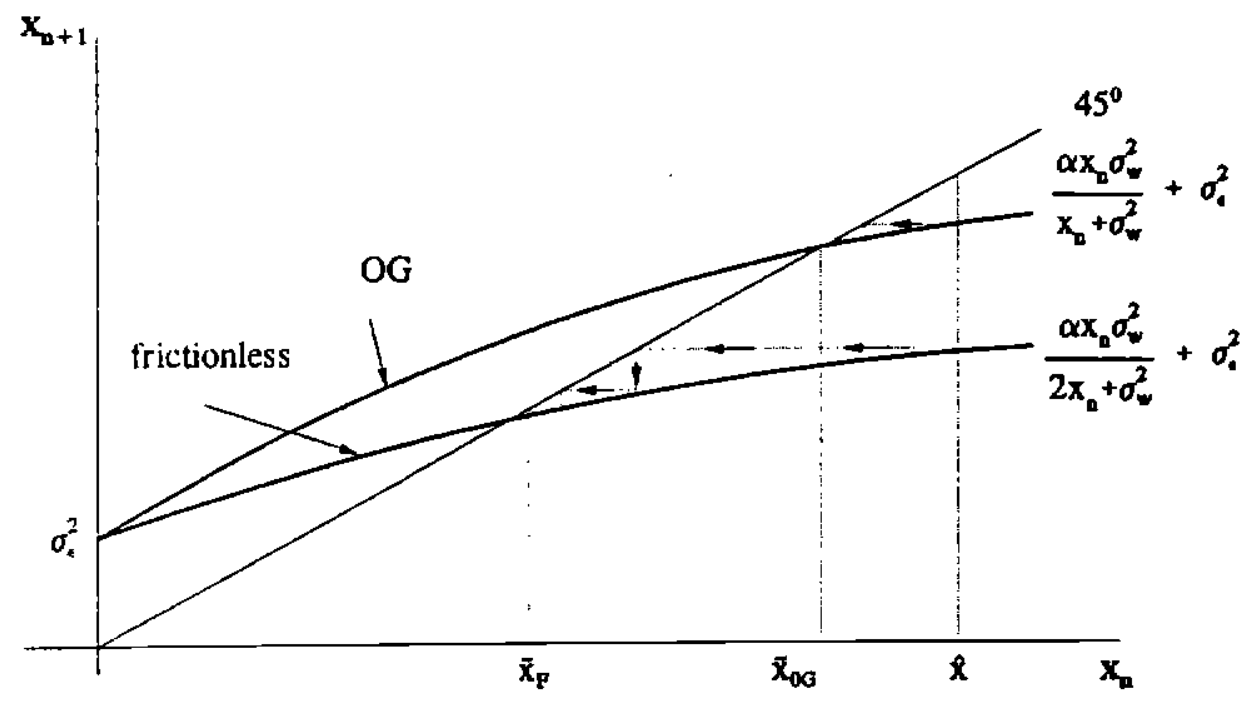

Figure 5: Evolution of $x_{n}$ in the two cases. 
Jovanovic and Nyarko The Transfer of Human Capital

\section{Productivity, Failure, and the Earning-Experience Profile}

8.1. Productivity and Failure If an old agent does not hire anyone, his technological line will end one period later. We define "failure" to be the cessation of hiring. In this model, less productive technologies are the more likely to fail. When $|m|<\bar{m}$, output per man of the two-agent team is

$$
\frac{1}{2}\left(1-x-a_{w}^{2} \cdot 1-x-o_{w}^{2}-m^{2}\right)=1-x-o_{w}^{2}-\frac{m^{2}}{2}=q_{a}(m)
$$

The probability that the firm will fail next period is

$$
Q_{n}(m)=\Phi\left(\frac{-\bar{m}_{n-1}-\alpha^{1 / 2 m}}{\alpha^{1 / 2} \sqrt{h_{1}\left(x_{n}\right) x_{n} / \sigma_{w}^{2}}}\right)+1-\Phi\left(\frac{\bar{m}_{n, 1}-\alpha^{1 / m_{m}}}{\alpha \sqrt{h_{1}\left(x_{n}\right) x_{n} / \sigma_{w}^{2}}}\right)
$$

where $\Phi$ is the normal cumulative distribution function. The function $Q_{0}(m)$ is increasing in $|m|$. The relevant values of $|\mathrm{m}|$ are those below $\overline{\mathrm{m}}$, and here $\mathrm{q}_{\mathrm{n}}$ and $\mathrm{Q}_{\mathrm{n}}$ are negatively related as $|\mathrm{m}|$ varies. This is shown in figure 6 . This implication fits the stylized fact that less productive firms are more likely to fail. The economics is that such firms fail because they find themselves in technologies that young agents find hard to assimilate; this may well be an important cause of failure in high-tech industries.

[figure 6 about here]. 


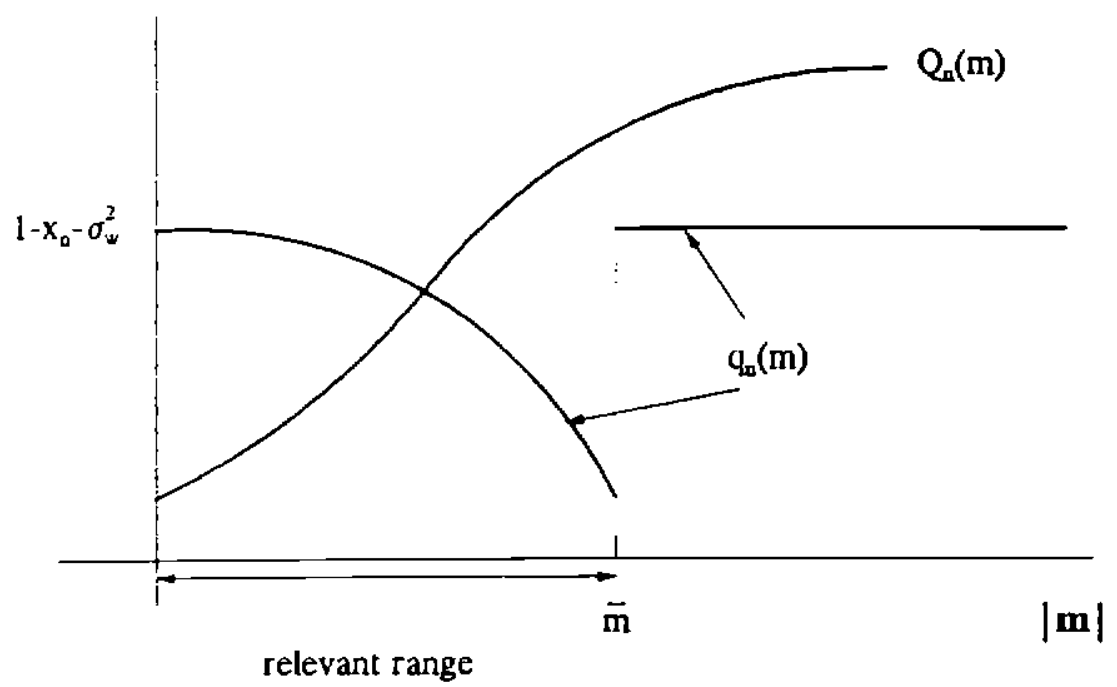

Figure 6: The negative relation between $\mathrm{a}_{n}$ and $Q_{n}$. 
Jovanovic and Nyarko The Transfer of Human Capial

8.2. Human Capital and the Earnings - Experience Profile. Old agents who operate older technological lines have higher productivity because they have a lower $x_{n}=B^{n}(\hat{x})$. When hired to work on an age-n technology, a young agent receives a level of consumption that (in view of (17) and (24)) equals

$$
c_{n}=u_{0}-\gamma \delta E_{2} V_{n \cdot 1}(m)
$$

When old, this agent's expected consumption will be $E_{2} V_{n+1}(m)$. Hence the age-expected earnings profile of this agent will have slope

$$
\frac{E_{1} V_{n-1}(m)}{c_{n}}=\left[\frac{u_{0}}{E_{0} V_{n-1}(m)}-\gamma \delta\right]^{\prime}
$$

On the right-hand side, only $V_{n+1}$ depends on $n$, and so the slope of the age-earnings profile will be relatively high in an age-n technology if its $V_{n+1}$ is relatively high.

\section{Conclusion}

We have modelled training, learning by doing, and production as joint activities. Training, however, occurs only if a young agent works togethei with an older one, and it consists of the younger agent watching the older one do the job. In equilibrium, some young agents start new firms on their own, while others join an older agent as apprentices. Apprentices give up part of their output in return for the training that they get from older agents, training that will raise their second period productivity.

The overlapping generations model differs radically from the single agent infinite horizon model studied in Jovanovic and Nyarko (1994). First, there is an avoidable adjustment cost -modelled as waiting time -- in the process by which knowledge is transferred from the old to the 
Sovanovic and Nyarko The Transter of Human Capital

young. This leads to a permanently lower productivity in the overlapping generations model. Second, the non-Ponzi equilibrium is constrained inefficient, in that too few new startups take place, and too many old technologies are kept alive. This is because the informational asymmetry between the old and the young gives rise to an adverse selection problem: an older agent with inferior skills pays his apprentice a wage that reflects not his own skills, but rather the skills of the average old agent.

\section{Appendix A: Construction and Properties of Equilibria.}

In the construction below, the reader may find figure 7 useful. First, we provide a summary of what is to come. We first define by induction a sequence of non-negative numbers $\left\{\Gamma_{0}\right\}_{n-1}^{\infty}$ and a sequence of functions $G_{n}:\left[\Gamma_{n-1}, \infty\right) \rightarrow \mathbb{R}$ for $n=1,2, \ldots$. We will then define for each $g_{1}>0$ sufficiently large, the sequence of numbers $\left\{g_{n}\right\}_{n=1}^{\infty}$ by setting $g_{n}=G_{n}\left(g_{1}\right)$. This sequence of numbers will then be shown to define an equilibrium. In particular, we obtain a multiplicity of equilibria with each equilibrium indexed by $g_{1}$. Now the details!

For each $\mathrm{g}_{1}>0$ define

$$
U_{1}\left(g_{1}\right)=\delta \gamma E_{1}\left[\operatorname{Max}\left\{0, g_{1}-m_{1}{ }^{2}\right\}\right]
$$

where the expectation $E_{1}$ is over the distribution of $m_{1}$. Define for each $n=1,2, \ldots$,

$$
\begin{aligned}
& h_{n}=\hat{x}-x_{n}+8 \gamma\left\{\hat{x}-x_{n+1}\right\} \text { and } \\
& H_{n}\left(g_{1}\right)=h_{n}-U_{1}\left(g_{1}\right) .
\end{aligned}
$$

Note that for each $n, H_{n}(0)>0, H_{n}\left(g_{1}\right)$ is strictly decreasing in $g_{1}$ and $H_{n}\left(g_{1}\right) \rightarrow-\infty$ as $g_{1} \rightarrow \infty$.

Define $\Gamma_{0} \equiv 0$ and $G_{1}\left(g_{1}\right) \equiv g_{1}$ on $\left[\Gamma_{0}, \infty\right)$. We proceed by induction. Suppose that for some $N \geq 1$ we have defined the non-negative numbers $\Gamma_{0}, \Gamma_{1}, \ldots, \Gamma_{N-1}$ and the functions $G_{n}:\left[\Gamma_{n-1}, \infty\right) \rightarrow R$ for 
Sovanovic and Nyarko The Transfer of Human Capital

$n=1, \ldots, N$, with $G_{n}\left(g_{1}\right)$ strictly increasing, taking the value 0 at $g_{1}=\Gamma_{n-1}$ and $\infty$ at $g_{1}=\infty$. Note that we have already shown this for $N=1$. Define $\Gamma_{N}>0$ be the unique number such that $G_{N}\left(g_{1}\right)-H_{N}\left(g_{1}\right)=0$ at $g_{1}=\Gamma_{N}$ and $G_{M}\left(g_{1}\right)-H_{N}\left(g_{1}\right)>0$ at each $g_{1}>\Gamma_{N}$. From the properties of $H_{N}$ and $G_{N}$ such a $\Gamma_{N}>0$ exists. Define $\mathrm{U}_{\mathrm{N}+1}: \mathbb{R}_{+}^{N^{N}} \mathbb{R}_{+} \rightarrow \mathbb{R}$ by

$$
U_{N+1}\left(g_{N+1}, g_{1}\right)=\delta \gamma E_{N+1}\left[\operatorname{Max}\left\{0, g_{N+1}-m_{N+1}^{2}\right\} \mid A_{1}, \ldots, A_{N}\right]
$$

where the expectation above is with respect to the distribution of $m_{\mathrm{N}+1}$ conditional on the events $A_{n}=\left\{m_{n} \in\left[-\left(G_{n}\left(g_{1}\right)\right)^{1 / 2},\left(G_{n}\left(g_{1}\right)\right)^{1 / 2}\right]\right\}$ for $n=1, \ldots, N$. For fixed $g_{1} \geq 0$, the function $U_{N+1}\left(., g_{1}\right)$ of $g_{N+1}$ is zero at $\mathrm{g}_{\mathrm{N}+1}=0$, and is strictly increasing taking the value $\infty$ at $\mathrm{g}_{\mathrm{N}+1}=\infty$. Define the function $G_{N+1}:\left[\Gamma_{N}, \infty\right) \rightarrow \mathbb{R}$ as follows: for each $g_{1} \geq \Gamma_{N}$ let $G_{N+1}\left(g_{1}\right)$ be the unique value of $g_{N+1}$ such that

$$
U_{N+1}\left(g_{N+1}, g_{1}\right)=G_{N}\left(g_{1}\right)-H_{N}\left(g_{1}\right)
$$

From the properties of the $U_{N+1}$ and $G_{N}\left(g_{1}\right)-H_{N}\left(g_{1}\right)$ just mentioned, it should be easy to see that the function $G_{N \cdot 1}\left(g_{1}\right)$ is well-defined on $\left[\Gamma_{N}, \infty\right)$, and is strictiy increasing taking the value 0 at $g_{1}=\Gamma_{N+1}$ and $\infty$ at $g_{1}=\infty$.

Hence by induction we have constructed a sequence of numbers $\left\{\Gamma_{n}\right\}_{n \rightarrow \infty}^{\infty}$ and a sequence of finctions $\left\{G_{n}\right\}_{n=1}^{\infty}$ with $G_{n}:\left[\Gamma_{n-1}, \infty\right) \rightarrow \mathbb{R}$ strictly increasing from 0 to $\infty$. Since by construction $\Gamma_{n}$ is the value of $g_{1}$ where $H_{n}\left(g_{1}\right)=G_{n}\left(g_{1}\right)$. As $n$ gets larger it should be clear (see fig. 7 ) that the functions $H_{n}$ and $G_{n}$ both shift to the right. Hence $\Gamma_{n-1}<\Gamma_{n}$ for all $n \geq 1$. Since $H_{n}\left(g_{1}\right)$ increases monotontically to $H_{x}\left(g_{1}\right) \equiv \lim _{n \rightarrow \infty} H_{n}\left(g_{1}\right)$, it should be clear that $\Gamma_{n} \leq H_{\infty}^{-1}(0)$ for all $n$. Hence $\lim _{n \rightarrow \infty}$ $\Gamma_{n}=\Gamma_{\infty}$ exists and is finite. Each of the functions $G_{0}\left(g_{1}\right)$ for $n \geq 1$ is therefore well-defined on $\left[\Gamma_{\infty}, \infty\right)$.

[Figure 7 about here]. 


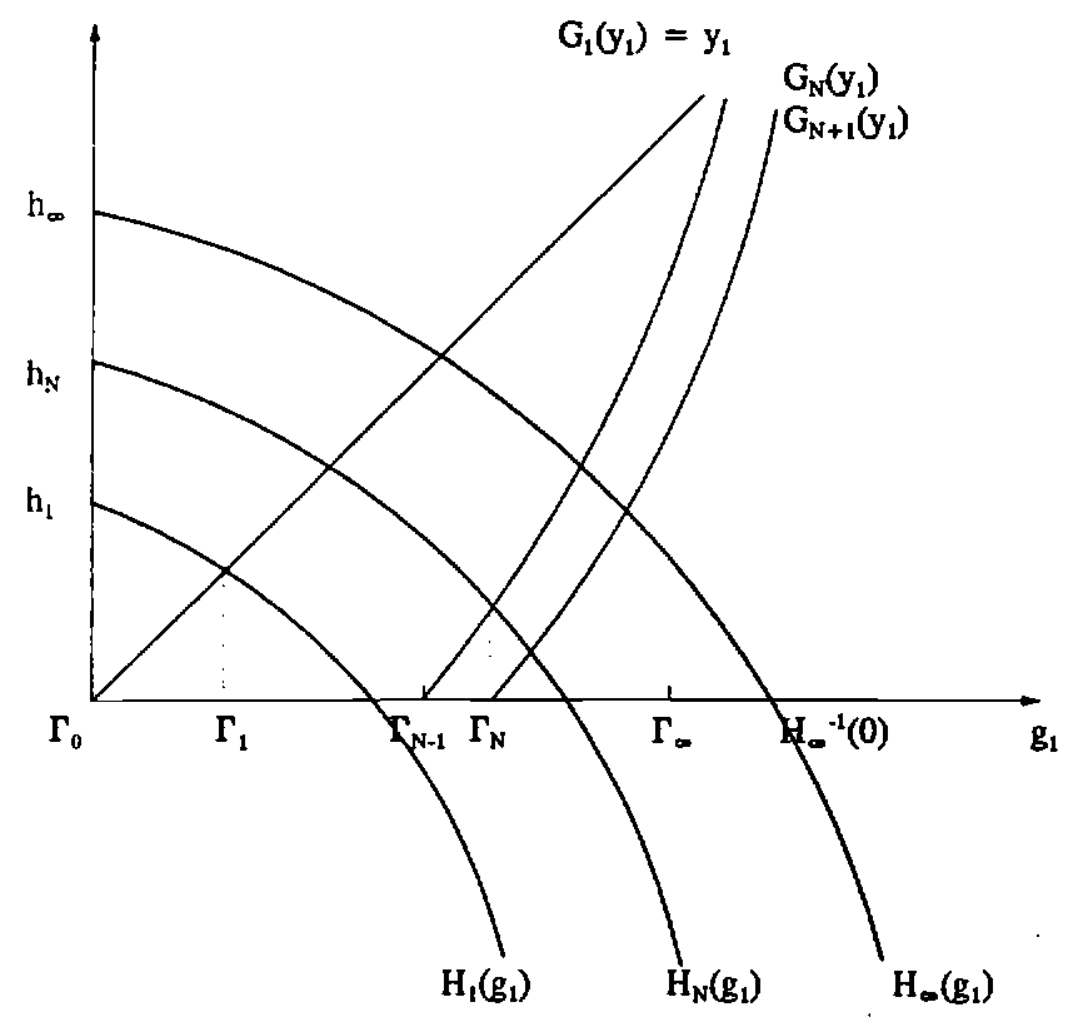

Figure 7: Construction of equilibrium. 
Sovanovic and Nyarko The Transfer of Human Capital

Fix any $g_{1} \geq \Gamma_{\infty}$. Define for each $n>0, \quad g_{a}=G_{n}\left(g_{1}\right)$ and $\bar{m}_{n}=g_{a}^{1 / 2}$. Define

$$
V_{n}(m)=1-\sigma_{w}^{2}-x_{n}+\operatorname{Max}\left\{0, g_{n}-m_{n}^{2}\right\} \quad \text { where } x_{n}=B^{n}\left(x_{n}\right)
$$

Equating (49) and (50) results in

$$
\begin{aligned}
& g_{N}=H_{N}\left(g_{1}\right)+U_{N+1}\left(g_{N+1}, g_{1}\right) \\
& =\hat{x}-x_{N}+\delta \gamma\left(\hat{x}-x_{N+1}+E_{N+1}\left[M a x\left\{0, g_{N+1}-m_{N+1}{ }^{2}\right\} \mid A_{1}, \ldots, A_{N}\right\}-E_{1}\left[M a x\left\{0, g_{1}-m_{1}{ }^{2}\right\}\right]\right\} \\
& =\hat{x}-x_{N}+\delta \gamma\left\{\left[1-\sigma_{w}{ }^{2}-x_{N+1}+E_{N+1}\left[\operatorname{Max}\left\{0, g_{N+1}-m_{N+1}{ }^{2}\right\} \mid A_{1}, \ldots, A_{N}\right]\right]-\left[1-\sigma_{w}{ }^{2}-\hat{x}+E_{1}\left[\operatorname{Max}\left\{0, g_{1}-m_{1}{ }^{2}\right\}\right]\right\}\right. \\
& \left.=\hat{x}-x_{N}+\delta \gamma\left\{E_{N+1}\left[V_{N+1}\left(m_{N+1}\right) \mid A_{1}, \ldots, A_{N}\right]\right]-E_{1}\left[V_{1}\left(m_{1}\right)\right]\right\} .
\end{aligned}
$$

Putting this in (51) gives

$$
\begin{aligned}
& V_{N}(m)=1-\sigma_{w}{ }^{2}-x_{N}+\operatorname{Max}\left\{0,1-\sigma_{w}{ }^{2}-x_{N}-m^{2}-c_{n}\right\} \quad \text { where } \\
& \left.c_{n} \equiv 1-\hat{x}-\sigma_{w}{ }^{2}-\delta \gamma\left\{E_{N+1}\left[V_{N+1}\left(m_{N+1}\right) \mid A_{1}, \ldots, A_{N}\right]\right]-E_{1}\left[V_{1}\left(m_{1}\right)\right]\right\}
\end{aligned}
$$

Since we may write $c_{n}$ above as

$$
c_{n}=u_{0}-\delta \gamma\left\{E_{N+1}\left[V_{N+1}\left(m_{N+1}\right) \mid A_{1}, \ldots, A_{N}\right]\right] \text { where } u_{0}=1-\hat{x}-\sigma_{w}^{2}+\delta \gamma E_{l}\left[V_{l}\left(m_{l}\right)\right]
$$

it should be clear that our construction satisfies the definition of an equilibrium. Hence each $g_{1}>\Gamma_{\infty}$ defines an equilibrium. 
Jovanovic and Vyarko The Transfer of Human Capital

Remarks:

K1: (Multiplicin of Equilibria) From the above construction we have a multiplicity of equilibria indexed by $g_{1}$ in the set $\left[\Gamma_{\infty,} \infty\right)$. Note that $g_{1}$ and $V_{1}(0)$ are related by the relation $V_{1}(0)=1-x_{1}-\sigma_{w}{ }^{2}$ $+\underline{s}_{1}$. When $g_{1}=\Gamma_{c}, V_{1}(0)=1-x_{1}-\sigma_{w}{ }^{2}+\Gamma_{\infty} \equiv \bar{v}$, say. Hence we could equivalently index the equilibria by the value of $V_{1}(0)$ so long as it lies in the set $[\bar{v}, \infty)$.

R2: (The Unique Bounded Infinite-Horizon Equilibrium): The equilibrium from $\mathrm{g}_{1}=\Gamma_{\infty}$ is such that if $V_{n}$ denotes the associated value functions then $\lim _{n \rightarrow \infty} V_{n}(m) \leq K$ for all $m$, where $K$ is a constant independent of $n$. (Indeed $K=1-\mathrm{x}_{\infty}-\sigma_{w}{ }^{2}+h_{\omega} /(1-\delta \gamma)$ ) This is the content of Proposition 10.1 below. We mention in the remark $R 3$ below that all equilibria from $g_{1}>\Gamma_{\infty}$ will have $\lim _{n \rightarrow \infty} V_{n}(m)=\infty$ for all $\mathrm{m}$. Hence the equilibrium associated with $\mathrm{g}_{1}=\Gamma_{\infty}$ is therefore referred to as the bounded equilibrium. Proposition 10.1 cbtains the bounded equilibrium via a construction which enables us to interpret it as the limit as $\mathrm{N} \rightarrow \infty$ of $\mathrm{N}$-horizon equilibria. The latter are equilibria where we insist that the decision problem is the same as the earlier analysis for all agents with technology of age $\mathrm{n} \leq \mathrm{N}+1$, but where we insist that no hiring is allowed on technologies of age $\mathrm{N}+1$ or larger. This equilibrium is obtained by setting $g_{1}=\Gamma_{n}$ and $g_{n}=G_{n}\left(g_{1}\right)$ for $n=1,2, \ldots, N+1$. ( In this case $g_{N+1}=0$ and $\mathrm{G}_{\mathrm{r}}\left(\mathrm{g}_{1}\right)$ is not well-detined for $\mathrm{r}>\mathrm{N}+1$.) There is a unique $\mathrm{N}$-horizon equilibrium of this type. The unique bounded equilibrium is the limit of the finite horizon equilibria as the horizon, $\mathrm{N}$, goes to intinity.

R3 The Ponzi Equilibrial: In proposition 10.2 below we show that for each equilibrium corresponding to i $g_{1}$ strictly greater than $\Gamma_{\infty}$ will have an unbounded limiting value function, i.e., $\lim _{n \rightarrow \infty} V_{n}(m)=\infty$ for all $n$. We refer to these equilibria as Ponzi equilibria.

\section{R4 (Ruling out Equilibria where there is never hiring on some gradel:}

Suppose that in an equilibrium there is some hiring on grade $n$ but no hiring on grade $n+1$. Hiring on grade $n$ (by the foreman of type $m_{n}=0$ ) implies that $1-x_{n}-\sigma_{w}{ }^{2} \geq c_{n} \quad$ (i.e., firms want to hire) and $u_{0}=c_{n}+\delta \gamma E_{a} V_{n+1}\left(m_{n+1}\right)=c_{n}+\delta \gamma\left[1-x_{n+1}{ }^{-} \sigma_{w}^{2}\right]$ (i.e., the young want to be hired) which implies that 
Jovanovic and Nyarko The Transter of Human Capital

$$
u_{0}-\delta \gamma\left[1-x_{n+1}-\sigma_{w}^{2}\right] \leq 1-x_{n}-\sigma_{w}^{2} .
$$

For there to be no hiring on grade $n+1,1-x_{n+1}-\sigma_{w}{ }^{2} \leq c_{n+1}$ and $u_{0} \geq c_{n+1}+\delta \gamma\left[1-x_{n+2}-\sigma_{w}{ }^{2}\right]$ which implies that $1-x_{p+1}-\sigma_{w}{ }^{2} \leq u_{0}-\delta \gamma\left[1-x_{n+2}-\sigma_{w}{ }^{2}\right]$. However since $x_{n+2}<x_{n+1}<x_{n}$, this is easily seen to be a contradiction to (52). Hence if there is hiring on grade $n$ there is also hiring on grade $n+1$.

It therefore remains only to show that there is hiring on grade 1. Suppose, per absurdem there is no hiring on grade 1 . Then it is easy to see that (52) holds for $n=0$ if we define $x_{0}=\hat{x}$. Mimicking the previous argument shows that assuming there is no hiring at date 1 leads to a contradiction to equation (52) with $n=0$. Hence in any equilibrium there is hiring on each grade.

Proposition 10.1. For all $n=1,2, \ldots, G_{n}\left(\Gamma_{\infty}\right)<h_{\sigma} /(1-\delta \gamma)<\infty$. In particular, if $\left\{\bar{m}_{1}, V_{1}\right\}_{n=1}^{\infty}$ is the equilibrium associated with $g_{1}=\Gamma_{\infty}$ as constructed above, then for each $m, \lim _{t \rightarrow \infty} V_{1}(m) \leq 1-x_{n}-\sigma_{w}{ }^{2}+$ $\mathrm{h}_{\delta} /(1-\delta \gamma)<\infty$.

Proof of Proposition 10.1: For each $n$, it is easy to see that $G_{n}\left(g_{1}\right)$ is continuous on $\left[\Gamma_{1}, \infty\right)$. We will later on show that

$$
G_{n}\left(\Gamma_{N}\right) \leq h_{\infty} /(1-\delta \gamma) \text { for each } n \text { and } N \text { with } N>n
$$

The first part of this proposition therefore follows immediately from taking limits as $\mathrm{N} \rightarrow \infty$ in (53). The second part of the proposition follows immediately from the first part and the definition of $V_{\mathrm{a}}(\mathrm{m})$ in (51).

We now prove (53). Fix any $N<\infty$. Define $g_{1}=\Gamma_{N}$ and $g_{a}=G_{n}\left(g_{1}\right)$ for all $n=1,2, \ldots, N+1$. From the earlier construction we know that such $\mathbf{g}_{1}, \ldots, \mathrm{g}_{\mathrm{N}+1}$ is well-defined with $\mathrm{g}_{\mathrm{N}+1}=0$. Further,

$$
g_{n}=U_{n+1}\left(g_{n+1}, g_{1}\right)+H_{n}\left(g_{1}\right) \quad \text { for each } n=1, \ldots, N
$$


Jovanovic and Nyarko The Transfer of Human Capial

However, from the definition of $U_{n+1}$ in (49), $U_{n+1}\left(g_{n+1}, g_{1}\right) \leq \delta \gamma g_{n+1}$. Also from (48), $H_{n}\left(g_{1}\right) \leq h_{\infty}$. Hence (5t) becomes, $g_{11} \leq \delta \gamma g_{n+1}+h_{\infty}$ for all $n=1, \ldots, N$. This is equivalent to

$$
\mathrm{g}_{\mathrm{N}-\mathrm{r}} \leq \delta \gamma \mathrm{g}_{\mathrm{N}-(\mathrm{r}-1)}+\mathrm{h}_{\infty} \text { for all } r=0,1, \ldots, \mathrm{N}-1
$$

Define $\mathrm{q}_{\mathrm{r}} \equiv \mathrm{g}_{\mathrm{N}-\mathrm{r}-1}$ for all $r=0,1, \ldots, \mathrm{N}-1$. Then (55) becomes

$$
q_{r+1} \leq \delta \gamma q_{r}+h_{\infty} \text { for all } r=0,1, \ldots, N-1
$$

Note that $q_{0}=g_{N+1}=0$. Define the linear function $f: \mathbb{R} \rightarrow \mathbb{R}$ by $f(q)=(\delta \gamma) q+h_{\infty}$. Iterates under this map from the origin will always remain below the fixed point, $h_{\infty} /(1-\delta \gamma)$, of this mapping. Since the right hand side of $(56)$ is $\mathrm{f}\left(\mathrm{q}_{\mathrm{r}}\right)$, we conclude that $\mathrm{q}_{\mathrm{r}} \leq \mathrm{h}_{\infty} /(1-\delta \gamma)$ for all $=0,1, \ldots, \mathrm{N}-1$. This in turn implies (53).

Proposition 10.2.: Fix any $g_{1}$ strictly greater than $\Gamma_{\infty}$. Then $\lim _{1-\infty} G_{1}\left(g_{1}\right)=\infty$. In particular, if $\left\{\bar{m}_{1}, V_{1}\right\}_{1-i}^{\infty}$ is the equilibrium associated with any $g_{1}>\Gamma_{\infty}$ as constructed above, then for each $m$, $\lim _{1 \rightarrow \infty} V_{1}(m)=\infty$.

Proof of Proposition 10.2.: The second part of the proposition follows immediately from the first part and the definition of $V_{n}(m)$ in (51). We now prove the first part of the proposition. We will later on show that

$$
\mathrm{dG}_{\mathrm{n}}\left(\mathrm{g}_{1}\right) / \mathrm{dg}_{1} \geq 1 /(\delta \gamma)^{\mathrm{n}} \quad \text { for all } \mathrm{n} \geq 1 \text { and at each } \mathrm{g}_{1} \geq \Gamma_{\mathrm{o}-1} \cdot
$$

Fix any $g_{1}>\Gamma_{\infty}$. (57) implies that for all $n, G_{n}\left(g_{1}\right)-G_{0}\left(\Gamma_{\infty}\right) \geq\left(g_{1}-\Gamma_{\infty}\right) /(\delta \gamma)^{n}$. Taking limits as $n \rightarrow \infty$ therefore implies that $\lim _{n \rightarrow \infty} G_{n}\left(g_{1}\right)=\infty$. Since $V_{a}(m)=1-\sigma_{w}^{2}-x_{n}+\operatorname{Max}\left\{0, g_{0}-m^{2}\right\}$, this implies that $V_{n}(m) \rightarrow \infty$

We now prove (57) by induction. Since $G_{1}\left(g_{1}\right)=g_{1}$ we see that the claim holds for $n=1$. So 
Jovanovic and Nyorko The Trunsfer of Human Capital

assume that (57) is true for some $n$. Putting $g_{a}=G_{a}\left(g_{1}\right)$ in (50) implies that

$$
U_{n+1}\left(G_{n+1}\left(g_{1}\right), g_{1}\right)=G_{0}\left(g_{1}\right)-H_{n}\left(g_{1}\right)
$$

Totally differentiating this with respect to $g_{1}$ and using the fact that $H_{n}$ is decreasing in $g_{1}$ implies that

$$
\left(d U_{n+1} / d g_{n+1}\right)\left(d G_{n+1} / d g_{1}\right)+\left(d U_{n+1} / d_{1}\right)=d G_{n} / d g_{1}-d H_{n} / d g_{1}
$$

Using the induction hypothesis and the fact that $\mathrm{dH}_{\mathrm{n}} / \mathrm{dg}_{1}<0$ we conclude that

$$
\left(d U_{n+1} / d g_{n+1}\right)\left(d G_{n+1} / d g_{1}\right)+\left(d U_{n+1} / d g_{1}\right) \geq l /(\delta \gamma)^{n}
$$

We will later show that for all $\mathrm{n}$,

$$
0<\mathrm{dU}_{\mathrm{n}+1} / \mathrm{dg}_{\mathrm{n}+1} \leq \delta \gamma \text { and } \mathrm{dU}_{\mathrm{n}+1} / \mathrm{dg}_{1}<0
$$

Purting (61) into (60) implies that $d G_{n+1} / d g_{1} \geq 1 /(\delta \gamma)^{n+1}$ which is the induction step for $n+1$. Hence by induction this shows that (57) is true for all $n$.

We now prove (61). Fix any $n \geq l$. It should be clear from the definition of $U_{\mathrm{a}}$ in (49) that for fixed $g_{1}$, an increase in $g_{n}$ by one unit will increase $U_{n}$, but by no more than $\delta \gamma$ units. Hence $0<\mathrm{dU}_{\mathrm{n}+1} / \mathrm{dg}_{\mathrm{n} r 1} \leq 1 / \delta \gamma$. This proves the first part of (61). Now let us consider the effect of an increase in $g_{1}$ keeping $g_{n+1}$ fixed. For any $r=1, \ldots, n$, since $G_{r}\left(g_{1}\right)$ is increasing in $g_{1}$, an increase in $g_{1}$ has the effect of increasing the conditioning sets $A_{r}$. ' $t$ is easily shown that this implies that the variance of $m_{n}$ will increase, with its mean remaining equal to zero. This in turn will lead to a reduction in the value of the integral in (49) which defines $U_{0}$. In particular, an increase in $g_{1}$ heeping $\mathrm{g}_{n+1}$ fixed will result in a decrease in $\mathrm{U}_{\mathrm{n}+1}$, so $\mathrm{dU}_{\mathrm{n}} / \mathrm{dg}_{1}<0$. This proves the second part of (61). 
11. Appendix B: Justification for Assumption (A.2). One implication of this assumption is that each continuing technological lir.e will be upgraded in every period. In this appendix, we show that such upgrading will be myopically optimal for some parameter values. Since old agents are by assumption myopic, the term "myopically" refers to the young agents. In this sense, myopically optimal policies maximize the expected output of the old and the young combined, but they ignore the future value of the training that takes place during the period.

Assume that it is the old agent who decides on whether to switch or not switch, in addition to deciding whether to hire or not hire. Then we have the matrix of the total expected outputs of the two agents: ${ }^{2}$

\begin{tabular}{|c|c|c|}
\hline & Don't Hire & Hire \\
\hline No Switch & $1-x-\sigma_{w}^{2}+\gamma\left[1-\hat{x}-\sigma_{w}^{2}\right]$ & $1-x-\sigma_{w}^{2}+1-x-m^{2}-\sigma_{w}^{2}-2\left(1-x-\sigma_{w}^{2}\right)-m^{2}$ \\
\hline Switch & $\gamma\left[1-\alpha x-\sigma_{\epsilon}^{2}-\sigma_{w}^{2}+1-\hat{x}-\sigma_{w}^{2}\right]$ & $\begin{array}{l}\gamma\left[1-\alpha x-\sigma_{e}^{2}-\sigma_{w}^{2}+1-\alpha x-\sigma_{e}^{2}-m^{2}-\sigma_{w}^{2}\right] \\
=\gamma\left[2\left(1-\alpha x-\sigma_{\varepsilon}^{2}-\sigma_{w}^{2}\right)-m^{2}\right]\end{array}$ \\
\hline
\end{tabular}

If he doesn't hire, the old agent will prefer to switch if

$$
1-x-\sigma_{w}^{2}<\gamma\left[1-\alpha x-\sigma_{\epsilon}^{2}-\sigma_{w}^{2}\right]
$$

If he does hire, he will prefer to switch if

2 If the young agent goes it alone, it is always optimal for him to start out on the latest available technology. This is because $\hat{x}=\alpha \hat{x}+\sigma_{t}^{2}$ so that $\gamma\left[1-\alpha x-\sigma_{e}{ }^{2}-\sigma_{w}{ }^{2}\right]$ always exceeds $1-x-$ $\sigma_{w}{ }^{2}$. This fact has been incorporated into the $(1,1)$ box of the matrix. 
Jovanovic and Nyarko The Transfer of Human Capizal

$$
1-x-\sigma_{w}^{2}<\gamma\left[1-\alpha x-\sigma_{c}^{2}-\sigma_{w}^{2}\right]-\frac{(\gamma-1)}{2} m^{2}
$$

Clearly, if (63) is met, then (62) is as well. If (63) holds for some $\mathrm{m}^{2}$, then it holds for all $\mathrm{m}^{2}$ less than that value. The largest possible $\mathbf{m}^{2}$ that, conditional on switching, will maintain the match is the one that equates the output under going it alone to the output under the match:

$$
\begin{aligned}
(\overline{\mathrm{m}})^{2} & =2\left(1-\alpha \mathrm{x}-\sigma_{\epsilon}^{2}-\sigma_{w}^{2}\right)-\left(1-\alpha \mathrm{x}-\sigma_{\epsilon}^{2}-\sigma_{w}^{2}\right)-\left(1-\hat{\mathrm{x}}-\sigma_{\mathrm{w}}^{2}\right) \\
& =\hat{\mathrm{x}}-\alpha \mathrm{x}-\sigma_{\epsilon}^{2} \leq \hat{\mathrm{x}}-\sigma_{\epsilon}^{2}=\frac{\alpha \sigma_{\epsilon}^{2}}{1-\alpha} .
\end{aligned}
$$

Since $m^{2} \leq(\bar{m})^{2}$, substitution for $m^{2}$ in (63) leads to the inequality

$$
1-x-\sigma_{w}^{2}<\gamma\left(1-\alpha x-\sigma_{\varepsilon}^{2}-\sigma_{w}^{2}\right) \cdot \frac{(\gamma-1) \alpha \sigma_{c}^{2}}{2(1-\alpha)}
$$

When $1-\sigma_{w}{ }^{2}>0$, the term $\left(1-\alpha x-\sigma_{e}{ }^{2}-\sigma_{w}{ }^{2}\right)$ is strictly positive for small enough $\alpha$ and $\sigma_{e}{ }^{2}$. Then (64) will hold when $\alpha$ and $\sigma_{\varepsilon}{ }^{2}$ are small enough and/or when $\gamma$ is large enough. So we have proved:

Proposition 11.1: There exist parameter values under which it is myopically optimal for all agents to upgrade their technologies in each period. 
Jovanovic and Nyerko The Transfer of Human Capital

\section{Appendix C: The Proofs.}

Proof of Proposition 4.1.: Let $\phi$ be any density function of some random variable $\eta$ taking values on the real line. Suppose that $\phi$ is symmetric about the origin and suppose that $\phi(\eta)$ is strictly decreasing in $|\eta|$. Let $\Phi$ be the cumulative distribution function corresponding to $\phi$. Fix any $k>0$ and any $M \geq 0$. Then

$$
\operatorname{Prob}\left(\left\{(M+\eta)^{2} \leq k\right\}\right)=\operatorname{Prob}(\{-k+M \leq \eta \leq k+M\})=\Phi(k+M)-\Phi(-k+M)
$$

However, taking derivatives with respect to $M$ in (65) yields

$$
\partial / \partial M\left[\operatorname{Prob}\left(\left\{(M+\eta)^{2} \leq k\right\}\right)\right]=\phi(k+M)-\phi(-k+M)
$$

Under the assumptions on $\phi$ it is easy to see that for $k>0$ and $M>0, \phi(k+M)-\phi(-k+M)<0$. Hence we conclude that Prob $\left(\left\{(M+\eta)^{2} \leq k\right\}\right)$ is decreasing in $M$.

Let $\eta$ be normal with mean equal to zero and variance $\alpha h_{1}\left(x_{n}\right) x_{u} / \sigma_{w}{ }^{2}$. From (65) and (66), $m_{n+1}$ is a random variable whose distribution conditional on $m_{n}$ is the same as $\alpha^{1 / 2} m_{a}+\eta$. Fix any $k>0$. Then

$$
\operatorname{Prob}\left(\left\{m_{n+1}^{2} \leq k \mid m_{n}\right)=\operatorname{Prob}\left(\left\{\left(\alpha^{1 / 2} m_{n}+\eta\right)^{2} \leq k\right\} \mid m_{n}\right)\right.
$$

Setting $M=\alpha^{1 / 2} m_{n}$ in our earlier argument shows that (67) is decreasing in $m_{a}$. This proves part (a) of our proposition.

(b) This follows immediately from part (a).

(c) Let $f: \mathbb{R}_{+} \rightarrow \mathbb{R}$ be any strictly increasing function. Since conditional on $m_{\mathbf{a}}$ the distribution of $m_{\mathrm{a}+1}$ is independent of the acceptance sets, we may write 
Jovanovic and Nyarko The Transfer of Human Capital

$$
E_{2}\left[f\left(m_{n+1}^{2}\right)\right]=E_{2}\left[f\left(m_{n+1}^{2}\right) \mid A_{1}, \ldots, A_{n}\right]=E_{2}\left[E\left[f\left(m_{n+1}^{2}\right) \mid m_{n}\right] \mid A_{1}, \ldots, A_{d}\right],
$$

where the outer expectation in the last term above is the expectation operator over $m_{\mathrm{a}}$ conditional on the events $A_{1}, \ldots, A_{n}$. From part (a), the bigger is $m_{n}$ the stochastically larger is the distribution of $m^{2}{ }_{n+1}$. That distribution is therefore largest at $\bar{m}_{n}$, the largest value of $m_{n}$ in $A_{n}$. Hence from (68) we conclude that $E_{2}\left[f\left(m_{n+1}^{2}\right)\right] \leq E_{2}\left[f\left(m_{n+1}^{2}\right) ; m_{n}=\bar{m}_{n}\right]$. Since $f$ is an arbitrary strictly increasing function this implies the conclusion of the proposition as regards $\mathrm{m}_{\mathrm{p}+1}^{2}$. Similar arguments prove the conclusion involving $\left|m_{n+1}\right|$

Proof of Proposition 4.3:: From (29)

$$
\sigma_{n, 1}^{2}=\frac{\alpha h_{1}\left(x_{n}\right) x_{n}}{\sigma_{w}^{2}} \cdot \alpha \hat{\sigma}_{n}^{2}
$$

where $\hat{\sigma}_{n}^{2}$ is the post truncation variance of $m_{n}$. Since $\alpha \geq 0$, and $0 \leq \hat{\sigma}_{n}^{2} \leq \hat{\sigma}_{n}^{2}$, the claim follows.

Proof of Proposition 5.3. This follows immediately from taking limits as $n \rightarrow \infty$ in (20), and using the defining property of a Ponzi equilibrium.

Proof of Lemma 6.1: Define $C(\mathbf{N x R})=\{W: N \times \mathbb{R} \rightarrow \mathbb{R}$ such that $W$ is continuous and bounded $\}$ and 
Jovanovic and Nyarko The Transfer of Hurnan Capial

$C_{d}(N x R)=\{W$ in $C(\mathbb{N x})$ which are decreasing in $|m|\}$. Then we may consider (36) as definining for each $W$ in $C(\mathbb{N x} R)$ a unique function (TW):NxR $\rightarrow \mathbb{R}$. It is easy to check that when $W$ is continuous and bounded so is $T W$. Hence $T: C(N \times \mathbb{R}) \rightarrow C(N \times R)$. Further, $T$ is a contraction operator. It therefore has a unique fixed point in $C(\mathbf{N x R})$. That fixed point is the function $W(n, m)$ $=W_{n}(m)$. From Corollary 4.2 , it is easy to show that if $W$ lies in $C_{d}(N \times R)$ then so too does $T W$. Hence $T: C_{d}(N \times \mathbb{R}) \rightarrow C_{d}(N \times R)$. The contraction mapping theorem therefore shows that $W_{a}(m)$ is decreasing in $|\mathrm{m}|$.

Proof of Proposition 6.2: From (41) and (39),

$(\delta \gamma)^{-1}\left[\left(m_{n}^{* 1-}\left(\bar{m}_{n}\right)^{2}\right]=\left\{E\left[W_{n+1}^{c}\left(m_{n+1}\right) \bar{m}_{n}^{*}-E_{2}\left[W_{n+1}^{c}\left(m_{n+1}\right)\right]\right\}-\left\{E_{1} W_{1}^{*}\left(m_{1}\right)-E W_{1}^{c}\left(m_{1}\right)\right\}\right.\right.$

From $(\$ 2)$, this becomes

$$
(\delta \gamma)^{-1}\left[\left(m_{n}^{* 2 \cdot}\left(\bar{m}_{n}\right)^{2}\right] \leq E\left[W_{n+1}^{e}\left(m_{n+1}\right)\left[\bar{m}_{a}^{*}\right]-E_{d}\left[W_{n+1}^{e}\left(m_{n+1}\right)\right]\right.\right.
$$

If we let $A_{1}, \ldots, A_{n}$ denote the equilibrium hiring sets, then

$$
\left.E_{a}\left[W_{n+1}^{e}\left(m_{a+1}\right)\right]=E_{2}\left[W_{a+1}^{e}\left(m_{a+1}\right) \mid A_{1}, \ldots, A_{d}\right]=E\left[E\left[W_{n+1}^{c}\left(m_{a+1}\right) \mid m_{b}\right]\right] A_{1}, \ldots, A_{d}\right] .
$$

where expectations operator $E\left[\mid A_{1}, \ldots, A_{n}\right]$ is over $m_{2}$ conditional on the hiring sets $A_{1}, \ldots, A_{a}$, and $E\left[. \mid m_{n}\right]$ is that over $m_{n+1}$ conditional on $m_{n}$. From Corollary 4.2 , the distribution of $\left|m_{a+1}\right|$ conditional on $m_{n}$ is stochastically increasing in the absolute value of $m_{n}$. From lemma 6.1, $W_{n+1}(m)$ is decreasing in $|m|$. Since $A_{a}=\left[-\bar{m}_{n}, \bar{m}_{n}\right]$, this implies that the right hand side of $(71)$ is less than $E\left[W_{n+1}^{e}\left(m_{n+1}\right) \mid \bar{m}_{11}\right]$. Putting this in (70) implies that 
Jovanovic and Nyarko The Transfer of Human Capital

$$
(\delta \gamma)^{-1}\left[\left(m_{n}^{* 2} \cdot\left(\bar{m}_{n}\right)^{2}\right] \leq E\left[W_{n+1}^{e}\left(m_{n+1}\right)\right] m_{n}^{*}\right]-E\left[W_{n+1}^{e}\left(m_{n+1}\right) \mid \bar{m}_{n}\right]
$$

Suppose per absurdem that $m_{n}^{*}>\bar{m}_{n}$. Then the left hand side of (72) is positive. Further, Corollary 4.2 implies that the right hand side of (72) is negative. This is a contradiction. Hence $m_{a}{ }^{*}$ $\leq \bar{m}_{n}$. 
Jovanovic and Nyarko The Transter of Human Capital

\section{References}

Barron, John, Dan Black, and Mark Lowenstein, 1989, Job Matching and On-the-Job Training, Journal of Labor Economics 7, 1-29.

Chari, V.V., and Hugo Jlopenhayn, 1991, Vintage Human Capital, Joumal of Political Economy 99, $1142-1165$.

Jovanovic, Boyan, and Yaw Nyarko, 1994, The Bayesian Foundations of Learning by Doing, unpublished paper, New York University.

Prescott, Edward, and John Boyd, Dynamic Coalitions, 1987, Engines of Growth, American Economic Review 77, 63-67. 